\title{
ULTRAFAST STRUCTURAL CHANGES WITHIN A PHOTOSYNTHETIC
} REACTION CENTRE

Robert Dods $^{1 \dagger}$, Petra Båth ${ }^{1 \dagger}$, Dmitry Morozov ${ }^{2}$, Viktor Ahlberg Gagnér ${ }^{1}$, David Arnlund ${ }^{1}$, Hoi Ling Luk ${ }^{2}$, Joachim Kübel ${ }^{1}$, Michał Maj $^{1}$, Adams Vallejos ${ }^{1}$, Cecilia Wickstrand ${ }^{1}$, Robert Bosman $^{1}$, Kenneth R. Beyerlein ${ }^{3}$, Garrett Nelson ${ }^{4}$, Mengning Liang ${ }^{6}$, Despina Milathianaki ${ }^{6}$, Joseph Robinson $^{6}$, Rajiv Harimoorthy ${ }^{1}$, Peter Berntsen ${ }^{1,7}$, Erik Malmerberg ${ }^{8}$, Linda Johansson $^{1,9}$, Rebecka Andersson ${ }^{1}$, Sergio Carbajo $^{6}$, Elin Claesson ${ }^{1}$, Chelsie E. Conrad $^{5}$, Peter Dahl $^{1}$, Greger Hammarin ${ }^{1}$, Mark S. Hunter ${ }^{6}$, Chufeng $\mathrm{Li}^{4}$, Stella Lisova ${ }^{4}$, Antoine Royant ${ }^{10,11}$, Cecilia Safari ${ }^{1}$, Amit Sharma ${ }^{1}$, Garth J. Williams ${ }^{6}$, Oleksandr Yefanov ${ }^{3}$, Sebastian Westenhoff ${ }^{1}$, Jan Davidsson ${ }^{12}$, Daniel P. DePonte ${ }^{6}$, Sébastien Boutet ${ }^{6}$, Anton Barty ${ }^{3}$, Gergely Katona ${ }^{1}$, Gerrit Groenhof ${ }^{2}$, Gisela Brändén ${ }^{1}$, Richard Neutze ${ }^{1 *}$

\footnotetext{
${ }^{1}$ Department of Chemistry and Molecular Biology, University of Gothenburg, Gothenburg,
} Sweden.

${ }^{2}$ Nanoscience Center and Department of Chemistry, University of Jyväskylä, Jyväskylä, Finland.

${ }^{3}$ Centre for Free-Electron Laser Science, Deutsches Elektronen-Synchrotron DESY, Hamburg, Germany.

${ }^{4}$ Department of Physics, Arizona State University, Tempe, Arizona, USA.

${ }^{5}$ Department of Chemistry and Biochemistry, Arizona State University, PO Box 871604 , Tempe, AZ 85287-1604, USA.

${ }^{6}$ Linac Coherent Light Source, Stanford Linear Accelerator Centre (SLAC) National Accelerator Laboratory, Menlo Park, CA, USA. 
$25{ }^{7}$ Australian Research Council Centre of Excellence in Advanced Molecular Imaging, La

26 Trobe Institute for Molecular Science, La Trobe University, Melbourne 3086, Australia

$27{ }^{8}$ Molecular Biophysics and Integrated Bioimaging, Lawrence Berkeley National Laboratory,

28 Berkeley, CA, USA.

$29{ }^{9}$ The Bridge Institute, Department of Chemistry, University of Southern California, Los

30 Angeles, CA 90089-3303, USA.

$31{ }^{10}$ Institut de Biologie Structurale (IBS), Université Grenoble Alpes, CEA, CNRS, F-38000

32 Grenoble, France

$33{ }^{11}$ European Synchrotron Radiation Facility, F-38043 Grenoble, France.

$34 \quad{ }^{12}$ Department of Chemistry-Angström laboratory, Uppsala University, Uppsala, Sweden.

39 These authors contributed equally to this work.

*Corresponding author: richard.neutze@gu.se

41 
Photosynthetic reaction centres harvest the energy content of sunlight by transporting electrons across an energy transducing biological membrane. We use time-resolved serial femtosecond crystallography ${ }^{1}$ at an X-ray free electron laser $^{2}$ to observe lightinduced structural changes in the photosynthetic reaction centre of Blastochloris viridis on a time-scale of picoseconds. Structural perturbations are first centred upon the protein's special pair of chlorophyll molecules that are photo-oxidized by light. Electron transfer to the menaquinone acceptor on the opposite side of the membrane induces a movement of this cofactor in concert with lower amplitude protein rearrangements. These observations reveal how proteins utilize conformational dynamics to stabilize the charge separation steps of electron transfer reactions.

Our biosphere depends upon the electron transfer reactions of photosynthesis as a primary source of energy. Photosystems and photosynthetic reaction centres form a family of integral membrane protein complexes found in plants, algae, cyanobacteria and photosynthetic bacteria that convert the energy of a captured photon into a charge separated state. The photosynthetic reaction centre of the purple non-sulphur bacterium Blastochloris viridis $\left(\mathrm{RC}_{v i r}\right)$ contains three transmembrane subunits called $\mathrm{H}, \mathrm{L}$ and $\mathrm{M}$ and a periplasmic subunit $\mathrm{C}$. These subunits support four bacteriochlorophyll molecules (BCh), two bacteriopheophytin molecules $(\mathrm{BPh})$, a tightly bound menaquinone $\left(\mathrm{Q}_{\mathrm{A}}\right)$, a mobile ubiquinone $\left(\mathrm{Q}_{\mathrm{B}}\right)$, a single nonhaem iron and four haem co-factors (Fig. 1). Electron transfer reactions originate at a special pair (SP) of strongly interacting bacteriochlorophylls which in $\mathrm{Bl}$. viridis have an absorption maximum at $960 \mathrm{~nm}$. Photo-oxidation of the SP liberates an electron which is transferred to the active branch $\mathrm{BPh}_{\mathrm{L}}$ within a few picoseconds, is transferred to the tightly bound menaquinone $\left(\mathrm{Q}_{\mathrm{A}}\right)$ in less than a nanosecond, and is transferred to the mobile ubiquinone $\left(\mathrm{Q}_{\mathrm{B}}\right)$ in microseconds. $\mathrm{SP}^{+}$is reduced by subunit $\mathrm{C}$ and a second photo-oxidation event transfers a 
second electron to $\mathrm{Q}_{\mathrm{B}}{ }^{-}$, which is protonated from the cytoplasm and released into the membrane as ubiquinol $\left(\mathrm{H}_{2} \mathrm{Q}\right)$. Other proteins participate in a cyclic flow that returns electrons to subunit $\mathrm{C}$ and the net effect is that two protons are transported across an energytransducing membrane for every photon absorbed.

Electrons may tunnel between cofactors when they are separated by approximately $10 \AA$ or less. ${ }^{3}$ The primary electron transfer step from $\mathrm{SP}$ to $\mathrm{BPh}_{\mathrm{L}}$ occurs in $2.8 \pm 0.2 \mathrm{ps}^{4}$ over a distance of $10 \AA$ by means of a two-step hopping mechanism via the monomeric $\mathrm{BCh}_{\mathrm{L}}{ }^{5}$ and is more rapid than conventional Marcus theory. By contrast, the $9 \AA$ electron transfer step from $\mathrm{BPh}_{\mathrm{L}}$ to $\mathrm{Q}_{\mathrm{A}}$ has a single exponential decay time of $230 \pm 30 \mathrm{ps}^{6}$ which is consistent with conventional Marcus theory. Coherent nuclear motions ${ }^{7}$ and protein structural changes ${ }^{8}$ have been suggested to influence the initial charge-transfer reactions of photosynthesis yet the specific nature of these putative protein motions is unknown. Flash-freeze crystallographic trapping studies, ${ }^{9}$ time-resolved Laue diffraction ${ }^{10}$ and time-resolved serial femtosecond crystallography $^{11-14}$ (TR-SFX) have revealed structural changes in bacterial photosynthetic reaction centres ${ }^{9,10}$ and cyanobacterial photosystem $\mathrm{II}^{11-14}$ that occur on the late microsecond to millisecond time-scale, yet no time-resolved crystallographic studies on the time-scale of the primary charge separation reactions of photosynthesis have been reported.

Here we apply time-resolved serial femtosecond crystallography ${ }^{1}$ at an X-ray free electron laser (XFEL) to probe the ultrafast structural response of $\mathrm{RC}_{v i r}$ to light. We photo-excited the special pair with 150 fs pulses centred at $960 \mathrm{~nm}$ (Extended Data Fig. 1). X-ray pulses $40 \mathrm{fs}$ in duration were generated at the LCLS ${ }^{2}$ and were used to record diffraction patterns from tens of thousands of microcrystals for the time points $\Delta t=1 \mathrm{ps}, 5 \mathrm{ps}$ (two repeats), $20 \mathrm{ps}, 300 \mathrm{ps}$ (two repeats) and $8 \mu$ s after photoexcitation (Extended Data Table 1). The time point $\Delta t=1 \mathrm{ps}$ 
populates the photo-excited charge transfer state of the SP in which charge rearrangements have occurred within the bacteriochlorophyll dimer but are prior to the primary electron transfer step; $\Delta t=5$ and $20 \mathrm{ps}$ are after the initial charge-transfer step and SP is oxidized and $\mathrm{BPh}_{\mathrm{L}}$ is reduced; $\Delta t=300 \mathrm{ps}$ is longer than the time constant for electron transfer to $\mathrm{Q}_{\mathrm{A}}$ and menaquinone is reduced; and $\Delta t=8 \mu$ s corresponds to a meta-stable charge separated state.

Extended Data Fig. 2 presents overviews of the $\left|\mathrm{F}_{\mathrm{obs}}\right|^{\text {light }}-\left|\mathrm{F}_{\mathrm{obs}}\right|^{\text {dark }}$ isomorphous difference Fourier electron density maps ("light" corresponds to data collected from photo-activated microcrystals whereas "dark" corresponds to data collected from microcrystals that were not photo-activated) for all time points. Difference electron density features are visible above 4.0 $\sigma$ ( $\sigma$ is the root mean square electron density of the map) near SP for all time points and strong features associated with $\mathrm{Q}_{\mathrm{A}}$ are visible for $\Delta t \geq 300 \mathrm{ps}$ (Extended Data Table 2). In contradistinction with ultrafast TR-SFX studies of bacteriorhodopsin, ${ }^{15}$ photoactive yellow protein, ${ }^{16}$ rsEGFP $^{17}$ and bacterial phytochromes ${ }^{18}$ in which ultrafast structural changes are driven by the movements of atoms due to a photo-isomerization event, TR-SFX measurements of $\mathrm{RC}_{v i r}$ reveal a knock-on effect on protein structure due to the light-induced redistribution of charge. Electric-field induced conformational changes have been observed when fields of the order $10^{8} \mathrm{~V} / \mathrm{m}$ are applied across a protein-crystal ${ }^{19}$ and this is the same order of magnitude as electric field perturbations due to the movement of an electron within $\mathrm{RC}_{v i r}$.

Recurring changes of electron density are visible as positive difference electron density in the region of overlap between the two bacteriochlorophylls $\mathrm{SP}_{\mathrm{L}}$ and $\mathrm{SP}_{\mathrm{M}}$ of the special pair, and complementary negative difference electron density features are visible primarily associated with $\mathrm{SP}_{\mathrm{M}}$ (Fig. 2, Extended Data Fig. 3, Extended Data Table 2, Supplementary Video 1). 
117 Singular value decomposition (SVD) of all seven difference Fourier electron density maps

118 (Fig. 2e) reveals that the strongest positive and several of the strongest negative difference

119 electron density features of the principal SVD component are associated with the SP

120 (Extended Data Table 2). Quantification of electron density changes ${ }^{20}$ within the $\mathrm{RC}_{v i r}$ co-

121 factors (Fig. 2f) and statistical tests against control difference Fourier electron density maps

122 (Methods) provides a very high-level of confidence ( $p$-value $\leq 0.001$, Extended data Table 3)

123 that these recurring difference electron density features do not arise by chance. Thus

124 photoexcitation causes the bacteriochlorophylls of SP to move closer together and the bending

125 (an out of plane distortion) of $\mathrm{SP}_{\mathrm{M}}$ could explain these observations. An out of plane

126 distortion was used to model difference electron density features observed as carbon

127 monoxide was photo-dissociated from the haem of myoglobin ${ }^{21}$ (Extended Data Fig. 4).

128 Nonplanar distortions of chlorin and bacteriochlorin rings are observed in photosystem II and

$129 \mathrm{RC}_{s p h}$. due to interactions with the surrounding protein ${ }^{22}$ and nonplanar porphyrins are also

130 more easily oxidized than planar porphyrins..$^{23,24}$ This suggests that the distortion of SP in

131 advance of the primary charge separation event (Fig. 2a) could enhance the yield of the

132 primary charge-transfer reaction, which has been optimized by evolution to achieve almost

133 perfect quantum efficiency. ${ }^{25}$

135 When the C-subunit is fully reduced, an electron is transferred from haem ${ }_{3}$ to $\mathrm{SP}^{+}$in less than

136 a microsecond. ${ }^{26}$ The above reasoning implies that $\mathrm{SP}^{+}$may be more easily reduced should

$137 \mathrm{SP}_{\mathrm{M}}$ return to a planar geometry before this electron transfer occurs. This hypothesis is

138 consistent with our experimental observations since the amplitude of the positive difference

139 electron density feature between the SP bacteriochlorophylls increases from $\Delta t=1$ ps to 20

$140 \mathrm{ps}$, decreases for $\Delta t=300 \mathrm{ps}$ and is insignificant for $\Delta t=8 \mu \mathrm{s}$ (Fig. 2, Extended Data Fig. 3,

141 Extended Data Table 2). Moreover, neither TR-SFX studies of the S2 $\rightarrow$ S3 transition of 
142 cyanobacteria $\operatorname{PSII}^{13}(\Delta t=150 \mu \mathrm{s}$ and $400 \mu \mathrm{s})$ nor TR-Laue diffraction studies of $\mathrm{RC}_{\mathrm{vir}}^{10}(\Delta t=$

$1433 \mathrm{~ms}$ ) report a positive difference electron density feature in the region of overlap between the 144 special pair of (bacterio)chlorophylls, implying that this feature has decayed.

Charge rearrangements cause $\mathrm{SP}^{+}$to move up to $0.3 \AA$ towards the M-subunit by $\Delta \mathrm{t}=300 \mathrm{ps}$ and the side-chains of both His $173_{\mathrm{L}}$ and His $200_{\mathrm{M}}$ adjust to preserve their ligating interactions with the magnesium ions of $\mathrm{SP}^{+}$, as do His $168_{\mathrm{L}}$ and $\mathrm{Tyr} 195_{\mathrm{M}}$ adjust conformation in order to maintain their $\mathrm{H}$-bond interactions to $\mathrm{SP}_{\mathrm{L}}$ and $\mathrm{SP}_{\mathrm{M}}$ respectively. These structural perturbations are revealed by paired negative and positive difference electron density features on the sidechain of His $173_{\mathrm{L}}$ in the principal SVD components calculated from both the early $(1 \mathrm{ps}, 2 \times$ $5 \mathrm{ps}, 20 \mathrm{ps})$ and late $(2 \times 300 \mathrm{ps}, 8 \mu \mathrm{s})$ sub-sets of TR-SFX data, whereas positive difference noticeably stronger for the later sub-set of data (Extended Data Fig. 3i,j; Extended Data Table 2; Supplementary Video 1). These observations suggest that $\mathrm{SP}_{\mathrm{L}}$ moves towards subunit $\mathrm{M}$ slightly in advance of $\mathrm{SP}_{\mathrm{M}}$, which may be due to dielectric asymmetry within photosynthetic reaction centres. ${ }^{27,28}$ Dielectric asymmetry is believed to underpin the phenomenon that electron transfer occurs only along the A-branch ${ }^{27}$ in purple bacteria RCs and PSII (Fig. 1).

An electron moves from $\mathrm{SP}$ to $\mathrm{BPh}_{\mathrm{L}}$ in $2.8 \pm 0.2 \mathrm{ps}^{4}$ and from $\mathrm{BPh}_{\mathrm{L}}$ to $\mathrm{Q}_{\mathrm{A}}$ in $230 \pm 30 \mathrm{ps}^{6}$ The tightly bound menaquinone is therefore neutral for $\Delta t=1 \mathrm{ps}, 5 \mathrm{ps}$ and $20 \mathrm{ps}$; threequarters of the photo-activated population are reduced to semiquinone by $\Delta t=300 \mathrm{ps}$; and essentially all photo-activated molecules have $\mathrm{Q}_{\mathrm{A}}$ reduced at $\Delta t=8 \mu \mathrm{s}$. Our difference Fourier electron density maps confirm these expectations since the few difference electron density features visible within the $\mathrm{Q}_{\mathrm{A}}$ binding pocket for $\Delta t \leq 20 \mathrm{ps}$ are isolated whereas more continuous paired positive and negative difference electron density features are visible for $\Delta t$ 
$167 \geq 300$ ps (Fig. 3, Extended Data Fig. 5). These recurring features of the later sub-set of TR-

168 SFX data $(2 \times 300 \mathrm{ps}, 8 \mu \mathrm{s})$ produce strong difference electron density features in the 169 principal SVD component that are associated with $\mathrm{Q}_{\mathrm{A}}$ and its $\mathrm{H}$-bond interaction with 170 His $217_{\mathrm{M}}$ (Extended Data Table 2, Fig. 3d, Supplementary Video 2) and statistical tests 171 establish that these recurring changes cannot be ascribed to noise ( $p$-value $\leq 0.0125$, Extended 172 Data Table 3). Structural refinement models these observations as due to a twist and 173 translation of the semiquinone that brings the negatively charged head-group approximately $1740.2 \AA$ closer to the positive charge of the non-haem $\mathrm{Fe}^{2+}$ (Fig. 3f) and thereby stabilizes the 175 reduced form of this cofactor. This interpretation receives support from QM/MM calculations 176 that predict that the $\mathrm{Q}_{\mathrm{A}}$ to His $217_{\mathrm{M}} \mathrm{H}$-bond is shortened by $0.17 \AA$ when $\mathrm{Q}_{\mathrm{A}}$ is reduced 177 (Extended Data Fig. 6f) and suggest that semiquinone binding is stabilized by approximately

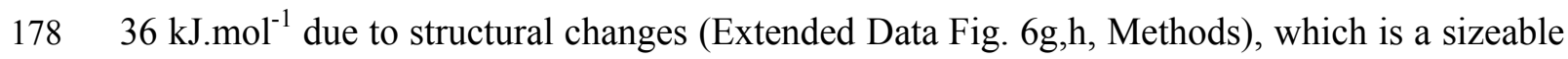
179 fraction of the energy $\left(125 \mathrm{~kJ} \cdot \mathrm{mol}^{-1}\right)$ of a $960 \mathrm{~nm}$ photon. Similar conclusions were drawn 180 from an earlier analysis using a Density Functional Theory (DFT) formalism. ${ }^{29}$ Light-induced 181 electron density changes were visible for $\mathrm{Q}_{\mathrm{A}}$ in TR-SFX studies of the $\mathrm{S} 2 \rightarrow \mathrm{S} 3$ transition of cyanobacteria PSII ${ }^{13}$ for the time points $150 \mu$ s and $400 \mu \mathrm{s}$, light-induced movements of the mobile quinone $\mathrm{Q}_{\mathrm{B}}$ were also observed in $\mathrm{PSII}^{11-14}$ for delays of hundreds of milliseconds, and larger light-induced motions of $\mathrm{Q}_{\mathrm{B}}$ were reported in freeze-trapping studies of the Rhodobacter sphaeroides photosynthetic reaction centre. ${ }^{9}$

187 For $\Delta t=300 \mathrm{ps}$, paired negative and positive difference electron density features are associated with the cytoplasmic portions of TM helices $\mathrm{D}_{\mathrm{M}}$ and $\mathrm{E}_{\mathrm{M}}$ (Fig. 3e) and indicate that $\mathrm{RC}_{v i r}$ adjusts its structure in response to the movement of the semiquinone within the $\mathrm{Q}_{\mathrm{A}}$ binding pocket (Fig. 3f). A more quantitative analysis (Methods, Extended Data Fig. 7, 191 Supplementary Video 3) suggests that low-amplitude protein motions begin to arise already 
192 by $\Delta t=1$ ps (Fig. 4a) as observed in TR-SFX studies of $\mathrm{bR}^{15,20}$ and $\mathrm{Mb}: \mathrm{CO}^{21}$; the amplitude

193 of these motions increase with time and by $\Delta t=5$ ps larger displacements are observed near 194 the $\mathrm{SP}^{+}$and $\mathrm{BPh}_{\mathrm{L}}{ }^{-}$cofactors (Fig. 4b); and for $\Delta t=300$ ps protein conformational changes 195 extend throughout the A-branch of the electron transfer pathway from $\mathrm{SP}^{+}$to $\mathrm{Q}_{\mathrm{A}}{ }^{-}$(Fig. $4 \mathrm{c}$ ).

196 When the same representation is used to depict protein conformational changes predicted 197 from QM/MM calculations (Supplementary Video 4) almost no structural changes are 198 expected for the photo-excited charge transfer state (Fig. 4d); protein movements arise near 199 the charged cofactors in the $\mathrm{SP}^{+}: \mathrm{BPh}_{\mathrm{L}}{ }^{-}$charge-separated state (Fig. 4e); and structural changes 200 extend throughout the A-branch in the $\mathrm{SP}^{+}: \mathrm{Q}_{\mathrm{A}}^{-}$charge-separated state (Fig. 4f). These 201 findings demonstrate that $\mathrm{RC}_{v i r}$ is not a passive scaffold but rather low-amplitude protein 202 motions engage in a choreographed dance with electron movements taking the lead and 203 protein conformational changes following. Conversely, as the protein's structure adjusts to 204 stabilize these charge rearrangements the energetic barriers hindering the reverse electron 205 transfer reaction increase, thereby extending the lifetime of the change separated species and enhancing the overall efficiency of photosynthesis.

In Marcus theory the total potential energy of an electron donor and its surroundings must be equal to that of the electron acceptor and its surroundings if an electron is to tunnel from

210 donor to acceptor. ${ }^{3}$ Fluctuations in the organizational energy around protein cofactors are 211 therefore essential to facilitate electron transfer reactions. Efforts aimed at understanding how 212 protein conformational dynamics control the rates of electron transfer between cofactors ${ }^{8,30}$ 213 have been hampered by a lack of experimental tools that characterize protein structural 214 changes on the relevant time-scales. Our observations provide an experimental framework for 215 extending the standard description of electron transfer reactions in photosynthesis ${ }^{3}$ to 216 explicitly incorporate protein structural changes. Electron transfer reactions are ubiquitous in 
217 nature and therefore a more nuanced understanding of the interplay between protein structural 218 dynamics and the movement of electrons has far-reaching biochemical significance.

219

220 
Extended Data is available for this manuscript.

Supplementary Information is available for this manuscript.

224

Acknowledgements Use of the Linac Coherent Light Source (LCLS), SLAC National Accelerator Laboratory, is supported by the U.S. Department of Energy, Office of Science, Office of Basic Energy Sciences under Contract No. DE-AC02-76SF00515. Parts of the sample delivery system used at LCLS for this research was funded by the NIH grant P41GM103393, formerly P41RR001209. Complementary studies were performed at BL3 of SACLA with the approval of the Japan Synchrotron Radiation Research Institute (JASRI, proposal number 2018B8068). RN acknowledges funding from the European Commission Marie Curie Training Networks (X-Probe, NanoMem), the European Union's Horizon 2020 research and innovation programme (grant agreement No. 789030) and the Swedish Research Council (grant No. 2015-00560 and 349-2011-6485), the Swedish Foundation for Strategic Research (grant SRL10-0036), and the Knut and Alice Wallenberg Foundation (grant KAW 2012.0284, KAW 2012.0275 and KAW 2014.0275). GB acknowledges funding from the Swedish Research Council (grant 2017-06734) and the Swedish Foundation for Strategic Research (grant ID17-0060). GG and HLL acknowledge funding from the Academy of Finland (grants 290677 and 304455), the BioExcel CoE project funded by the European Union contracts H2020-INFRAEDI-02-2018-823830 and H2020-EINFRA-2015-1-675728, and the CSC-IT centre in Espoo for access to computing resources. R.N. wishes to thank Professors Neal Woodbury for discussions.

\section{Author Information}

These authors contributed equally: Robert Dods, Petra Båth. 


\section{Contributions}

248 R.N. conceived the experiment, which was designed with input from G.B., R.D., J.D., S.B., 249 M.L., S.C., D.M., M.S.H., G.J.W., J.D., D.P.D. and A.B. Samples were prepared by R.D., 250 P.Bå., D.A. and R.B. Crystallization was supervised by G.B.. Time-resolved serial 251 femtosecond crystallography experiments were performed at the LCLS by R.D., P.Bå., D.A., 252 R.B. K.R.B., G.N., M.L., D.M., J.R., R.H., P.Be., E.M., L.J., R.A., S.C., E.C., C.E.C., P.D., 253 G.H., M.S.H., C.L., S.L. C.S., A.S., G.J.W., C.W., J.D., D.P.D., A.B., G.B. and R.N. The CXI 254 instrument was set up and run by M.L., M.S.H., G.J.W. and S.B.. The photoexcitation laser of $255960 \mathrm{~nm}$ was operated and aligned by D.M., J.R., S.C. and J.D.. Sample delivery was 256 performed by K.R.B., G.N., R.H., P.Be., P.D. and D.P.D. Data were analysed by R.D., P.Bå., 257 A.R., O.Y., A.B., G.B. and R.N. Structural refinement was performed by R.D. and P.Bå. 258 Resampling, full occupancy structural refinement and analysis were performed by V.A.G, 259 G.K. and A.V. Integration within a sphere and statistical tests were performed by C.W., R.N. and P.Bå.. SVD analysis was performed by A.V. Quantum Mechanics/Molecular Mechanics analysis were performed by D.M., H.L.L and G.G.. Time-resolved IR spectroscopy measurements were performed by J.K, M.M and S.W. The manuscript was prepared by R.N., P.Bå., R.D. and G.B. with additional input from all authors.

\section{Corresponding Author}

266 Correspondence to Richard Neutze (richard.neutze@gu.se).

\section{Code Availability}

269 Software used for SVD analysis is available at https://github.com/Neutze-lab/SVD. Code 270 written in MATLAB to analyse difference electron density amplitudes is available at 
272 diffraction data is available at https://github.com/Katona-lab/CFEL tools.

274 Data Availability

275 Atomic coordinates and structure factors have been deposited in the Protein Data Bank, 276 www.pdb.org. PDB ID codes are the following: 5O4C the dark-conformation (a); 5NJ4 the 277 dark conformation (b); 6ZHW the time-point $\Delta t=1 \mathrm{ps} ; 6 \mathrm{ZI} 4$ the time-point $\Delta t=5 \mathrm{ps}$ (a); 278 6ZID the time-point $\Delta t=5$ ps (b); 6ZI6 the time-point $\Delta t=20 \mathrm{ps;6ZI5} \mathrm{the} \mathrm{time-point} \Delta t=$ $279300 \mathrm{ps}(\mathrm{a}) ; 6 \mathrm{ZI} 9$ the time-point $\Delta t=300 \mathrm{ps}(\mathrm{b}) ; 6 Z \mathrm{ZIA}$ the time-point $\Delta t=8 \mu \mathrm{s}$. Difference 280 Fourier electron density maps and stream files containing X-ray diffraction intensities are 281 deposited at the CXI database (http://www.cxidb.org/) with identification number ID 161.

\section{Competing Interests}

284 The authors declare no competing interests. 
2921 Tenboer, J. et al. Time-resolved serial crystallography captures high-resolution 293 intermediates of photoactive yellow protein. Science 346, 1242-1246 (2014).

2942 Emma, P. et al. First lasing and operation of an angstrom-wavelength free-electron 295 laser. Nat. Photon. 4, 641-647 (2010).

2963 Marcus, R. A. \& Sutin, N. Electron transfers in chemistry and biology. Biochim. 297 Biophys. Acta 811, 265-322 (1985).

2984 Breton, J., Martin, J. L., Migus, A., Antonetti, A. \& Orszag, A. Femtosecond 299 spectroscopy of excitation energy transfer and initial charge separation in the reaction 300 center of the photosynthetic bacterium Rhodopseudomonas viridis. Proc. Natl. Acad.

$301 \quad$ Sci. USA 83, 5121-5125 (1986).

3025 Chan, C. K., DiMagno, T. J., Chen, L. X., Norris, J. R. \& Fleming, G. R. Mechanism 303 of the initial charge separation in bacterial photosynthetic reaction centers. Proc. Natl. $304 \quad$ Acad. Sci. USA 88, 11202-11206 (1991).

3056 Holten, D., Windsor, M. W., Parson, W. W. \& Thornber, J. P. Primary photochemical 306 processes in isolated reaction centers of Rhodopseudomonas viridis. Biochim. 307 Biophys. Acta 501, 112-126 (1978).

3087 Vos, M. H., Rappaport, F., Lambry, J.-C., Breton, J. \& Martin, J.-L. Visualization of 309 coherent nuclear motion in a membrane protein by femtosecond spectroscopy. Nature $310 \quad 363,320-325(1993)$.

3118 Wang, H. et al. Protein dynamics control the kinetics of initial electron transfer in 312 photosynthesis. Science 316, 747-750 (2007).

3139 Stowell, M. H. et al. Light-induced structural changes in photosynthetic reaction 314 center: implications for mechanism of electron-proton transfer. Science 276, 812-816 315 (1997). 
31610 Wöhri, A. B. et al. Light-induced structural changes in a photosynthetic reaction 317 center caught by Laue diffraction. Science 328, 630-633 (2010).

31811 Young, I. D. et al. Structure of photosystem II and substrate binding at room 319 temperature. Nature 540, 453-457 (2016).

32012 Suga, M. et al. Light-induced structural changes and the site of $\mathrm{O}=\mathrm{O}$ bond formation 321 in PSII caught by XFEL. Nature 543, 131-135 (2017).

32213 Kern, J. et al. Structures of the intermediates of Kok's photosynthetic water oxidation $323 \quad$ clock. Nature 563, 421-425 (2018).

32414 Suga, M. et al. An oxyl/oxo mechanism for oxygen-oxygen coupling in PSII revealed 325 by an x-ray free-electron laser. Science 366, 334-338 (2019).

32615 Nogly, P. et al. Retinal isomerization in bacteriorhodopsin captured by a femtosecond 327 X-ray laser. Science 361, eaat0094 (2018).

32816 Pande, K. et al. Femtosecond structural dynamics drives the trans/cis isomerization in 329 photoactive yellow protein. Science 352, 725-729 (2016).

33017 Coquelle, N. et al. Chromophore twisting in the excited state of a photoswitchable 331 fluorescent protein captured by time-resolved serial femtosecond crystallography. $332 \quad$ Nature Chem 10, 31-37 (2018).

33318 Claesson, E. et al. The primary structural photoresponse of phytochrome proteins 334 captured by a femtosecond X-ray laser. Elife 9, e53514 (2020).

33519 Hekstra, D. R. et al. Electric-field-stimulated protein mechanics. Nature 540, 400-405 $336 \quad$ (2016).

33720 Wickstrand, C. et al. A tool for visualizing protein motions in time-resolved $338 \quad$ crystallography. Struct Dyn 7, 024701 (2020).

33921 Barends, T. R. et al. Direct observation of ultrafast collective motions in CO $340 \quad$ myoglobin upon ligand dissociation. Science 350, 445-450 (2015). 
34122 Saito, K. et al. Deformation of chlorin rings in the Photosystem II crystal structure.

$342 \quad$ Biochemistry 51, 4290-4299 (2012).

34323 Shelnutt, J. A. et al. Nonplanar porphyrins and their significance in proteins. Chem. $344 \quad$ Soc. Rev. $2731-42$ (1998).

34524 Barkigia, K. M., Chantranupong, L., SmithJ, K. M. \& Fajer, J. Structural and 346 theoretical models of photosynthetic chromophores. Implications for redox, light347 absorption properties and vectorial electron flow. J. Am. Chem. Soc. 110, 7566-7567 $348 \quad$ (1988).

34925 Wraight, C. A. \& Clayton, R. K. The absolute quantum efficiency of 350 bacteriochlorophyll photooxidation in reaction centres of Rhodopseudomonas 351 spheroides. Biochim Biophys Acta 333, 246-260 (1974).

35226 Dohse, B. et al. Electron transfer from the tetraheme cytochrome to the special pair in 353 the Rhodopseudomonas viridis reaction center: effect of mutations of tyrosine L162. $354 \quad$ Biochemistry 34, 11335-11343 (1995).

35527 Steffen, M. A., Lao, K. \& Boxer, S. G. Dielectric asymmetry in the photosynthetic 356 reaction center. Science 264, 810-816 (1994).

35728 Najdanova, M., Grasing, D., Alia, A. \& Matysik, J. Analysis of the Electronic 358 Structure of the Special Pair of a Bacterial Photosynthetic Reaction Center by (13) C 359 Photochemically Induced Dynamic Nuclear Polarization Magic-Angle Spinning NMR $360 \quad$ Using a Double-Quantum Axis. Photochem Photobiol 94, 69-80 (2018).

36129 Hasegawa, J.-Y. et al. Energetics of the Electron Transfer from Bacteriopheophytin to 362 Ubiquinone in the Photosynthetic Reaction Center of Rhodopseudomonas Viridis: 363 Theoretical Study. J. Phys. Chem. B 107, 838-847 (2003). 
36430 LeBard, D. N., Martin, D. R., Lin, S., Woodbury, N. W. \& Matyushov, D. V. Protein dynamics to optimize and control bacterial photosynthesis. Chem. Sci. 4, 4127-4136 (2013).

FIGURE LEGENDS

Figure 1: Electron transfer steps of the photosynthetic reaction centre of Bl. viridis.

Cartoon representation of the $\mathrm{H}, \mathrm{L}, \mathrm{M}$ and $\mathrm{C}$ subunits. Cofactors are shown in black including the special pair of bacteriochlorophylls (SP), two monomeric bacteriochlorophylls (BCh), two bacteriopheophytins $(\mathrm{BPh})$, a tightly bound menaquinone $\left(\mathrm{Q}_{\mathrm{A}}\right)$, a mobile ubiquinone $\left(\mathrm{Q}_{\mathrm{B}}\right)$, a non-haem iron $\left(\mathrm{Fe}^{2+}\right)$ and four haems. The approximate boundaries of the membrane are suggested in blue. The electron transfer pathway: $\mathrm{SP} \rightarrow \mathrm{BPh}_{\mathrm{L}} \rightarrow \mathrm{Q}_{\mathrm{A}}$ is referred to as the Abranch. Approximate timescales for the first two electron transfer events, from $\mathrm{SP}$ to $\mathrm{BPh}_{\mathrm{L}}$ and from $\mathrm{BPh}_{\mathrm{L}}$ to $\mathrm{Q}_{\mathrm{A}}$, are depicted.

Figure 2: Light-induced electron density changes in $\mathrm{RC}_{v i r}$ at the site of photo-oxidation. a, Experimental $\mathrm{F}_{\mathrm{obs}}{ }^{\text {light }}-\mathrm{F}_{\mathrm{obs}}{ }^{\text {dark }}$ difference Fourier electron density map for $\Delta t=1 \mathrm{ps}$. b, Difference Fourier electron density map for $\Delta t=5$ ps (data set a). c, Difference Fourier electron density map for $\Delta t=20 \mathrm{ps}$. d, Difference Fourier electron density map for $\Delta t=300$ ps (data set a). e, Principal component from SVD analysis of all seven experimental square electron density of the map). f, Relative amplitudes of difference electron density features integrated within a $4.5 \AA$ sphere ${ }^{20}$ centred upon the $\mathrm{RC}_{v i r}$ co-factors (Extended Data

Fig. $3 \mathrm{j}$ ). The colour bars represent: cyan, $\Delta t=1 \mathrm{ps}$; blue, $\Delta t=5 \mathrm{ps}$ a and b (in that order); purple, $\Delta t=20 \mathrm{ps}$; red, $\Delta t=300 \mathrm{ps}$ b and a (in that order); mustard, $\Delta t=8 \mu \mathrm{s}$. 
Figure 3: Light-induced electron density changes in $\mathrm{RC}_{v i r}$ within the menaquinone

391 binding pocket. a, Experimental $\mathrm{F}_{\mathrm{obs}}{ }^{\text {light }}-\mathrm{F}_{\mathrm{obs}}{ }^{\text {dark }}$ difference Fourier electron density map for $\Delta t=5 \mathrm{ps}$ (data set a). $\mathbf{b}$, Difference Fourier electron density map for $\Delta t=300$ ps (data set a).

c, Principal component from SVD analysis of the first four experimental difference Fourier electron density maps ( $\Delta t=1 \mathrm{ps}, 5 \mathrm{ps}(\mathrm{a}), 5 \mathrm{ps}(\mathrm{b}), 20 \mathrm{ps})$. d, Principal component from SVD analysis of the final three experimental difference Fourier electron density maps $(\Delta t=300 \mathrm{ps}$ (a), $300 \mathrm{ps}(\mathrm{b}), 8 \mu \mathrm{s}$ ). All maps are contoured at $\pm 3.0 \sigma$ (blue, positive difference electron density; gold, negative difference electron density). e, Difference Fourier electron density map for $\Delta t=300 \mathrm{ps}$ (data set a) showing the protein immediately surrounding $\mathrm{Q}_{\mathrm{A}}$ and contoured at $\pm 3.5 \sigma$. f, Superposition of the refined structures for the dark structure (yellow, $\mathrm{Q}_{\mathrm{A}}$ in black) and $\Delta t=300 \mathrm{ps}$ (purple structure).

Figure 4: Structural response of $\mathrm{RC}_{v i r}$ to electron transfer events. a, Recurring movements of $\mathrm{C} \alpha$ atoms for $\Delta t=1 \mathrm{ps}$ quantified by full occupancy structural refinement $\Delta t=5$ ps (a) using the same representation. $\mathbf{c}$, Recurring movements of $\mathrm{C} \alpha$ atoms for $\Delta t=300$ ps (a) using the same representation. Recurring movements are represented as error weighted mean ratios relative to 100 control structural refinements (Methods) coloured from grey $(<80$

$\%$ of the maximum error weighted mean ratio) to red ( $\geq 95 \%$ of the maximum error weighted mean ratio). An identical representation is given for all time points in Extended Data Fig. 7. d, Movements of $\mathrm{C} \alpha$ atoms estimated from $\mathrm{QM} / \mathrm{MM}$ energy minimization calculations 411 associated with the SP photo-excited and all other cofactors in resting state: $\mathrm{SP} *: \mathrm{BPh}_{\mathrm{L}}{ }^{0}: \mathrm{Q}_{\mathrm{A}}{ }^{0}$ 412 (Methods). e, Movements of $\mathrm{C} \alpha$ atoms estimated from QM/MM energy minimization 413 calculations associated with the SP photo-oxidized and $B P h_{L}$ reduced: $\mathrm{SP}^{+1}: \mathrm{BPh}_{\mathrm{L}}{ }^{-1}: \mathrm{Q}_{\mathrm{A}}{ }^{0} . \mathbf{f}$, 
414 Movements of $\mathrm{C} \alpha$ atoms estimated from $\mathrm{QM} / \mathrm{MM}$ energy minimization calculations

415 associated with $\mathrm{SP}$ photo-oxidized and $\mathrm{Q}_{\mathrm{A}}$ reduced: $\mathrm{SP}^{+1}: \mathrm{BPh}_{\mathrm{L}}{ }^{0}: \mathrm{Q}_{\mathrm{A}}{ }^{-1}$. Movements are 416 coloured from grey (no movements) to red (maximum $\mathrm{C} \alpha$ motions). Transmembrane helices 417 are drawn as rods. 


\section{Protein production and purification}

420 The expression and purification of photosynthetic reaction centre from $\mathrm{Bl}$. viridis cells was 421 adapted from Wöhri et al. ${ }^{31}$. Cells were disrupted by three rounds of sonication followed by centrifugation in a JA20 rotor at $15000 \mathrm{rpm}$ for 20 minutes to recover the membrane suspensions. Membranes were then purified by ultracentrifugation at $45000 \mathrm{rpm}$ for $45 \mathrm{~min}$ in a Ti45 rotor. Membranes were homogenized in $20 \mathrm{mM}$ Tris- $\mathrm{HCl}, \mathrm{pH} 8.5$ and diluted to $\mathrm{OD}_{1012}=10$. Membranes were then solubilized in $4 \%$ lauryldimehtylamine-N-oxide (LDAO) for 3 hours at room temperature. Unsolubilized membranes were removed by ultracentrifugation at $45000 \mathrm{rpm}$ for $75 \mathrm{~min}$ in a $\mathrm{Ti} 70$ rotor. $\mathrm{RC}_{v i r}$ protein was purified by loading the supernatant onto a $250 \mathrm{ml}$ POROS 50- $\mu \mathrm{m}$ HQ ion-exchange medium equilibrated with wash buffer (20 mM Tris-HCl, $\mathrm{pH} 8.5,1 \%$ LDAO). The column was washed with 21 of wash buffer with $5 \%$ elution buffer (20 mM Tris-HCL, pH 8.5, $1 \mathrm{M} \mathrm{NaCl}, 1 \%$ LDAO) and eluted with an increasing concentration of elution buffer over 20 column volumes. Fractions with an $\mathrm{A}_{280} / \mathrm{A}_{830}<3.5$ were pooled and concentrated in $100 \mathrm{kDa} \mathrm{MW}$ cut off concentration tubes (Vivaspin) to a volume of $10 \mathrm{ml}$. This was loaded in $5 \mathrm{ml}$ batches onto a HiPrep 26/60 Sephacryl S-300 column (GE) equilibrated with SE buffer (20 mM Tris-HCl, pH 8.5, $100 \mathrm{mM}$ $\mathrm{NaCl}, 0.1 \% \mathrm{LDAO}$ ) and eluted into $1.8 \mathrm{ml}$ fractions. Fractions with an $\mathrm{A}_{280} / \mathrm{A}_{830}<2.6$ were $\mathrm{mg} \cdot \mathrm{ml}^{-1}$. Samples were flash-frozen in liquid nitrogen and stored at $-80{ }^{\circ} \mathrm{C}$.

Protein crystallization

$44120 \mu \mathrm{l}$ sitting drops were set up with a 1:1 ratio of protein solution $\left(10 \mathrm{mg} \cdot \mathrm{ml}^{-1}\right)$ and precipitant 442 solution (3.6 M ammonium sulphate, $6 \%$ heptane-1,2,3-triol, $20 \mathrm{mM} \mathrm{NaH} \mathrm{PO}_{4} / \mathrm{Na}_{2} \mathrm{HPO}_{4}, \mathrm{pH}$ 
6.8) set up against a $1 \mathrm{ml}$ reservoir of $2 \mathrm{M}$ ammonium sulphate. Large crystals grew at $4{ }^{\circ} \mathrm{C}$ in 3 days. Crystals were harvested by pipette and crushed mechanically to create a seed stock by vortexing with seed beads for approximately 20 min with occasional cooling on ice ${ }^{32}$. For the XFEL experiment in April 2015 (run a) new $18.5 \mu 1$ sitting drop vapour diffusion crystallization drops were set up in order to yield large numbers of micro-crystals. In these experiments the protein concentration was $8.5 \mathrm{mg} \cdot \mathrm{ml}^{-1}$ and a protein:precipitant concentration of 10:7.5 was used in the drops. $1 \mu \mathrm{l}$ of undiluted crystal seed stock was spiked into the drops for a final $\mathrm{v} / \mathrm{v}$ concentration of $5.4 \%$. Crystallization drops were then mixed by pipette and covered with a glass cover slide. Rod-like crystals grew over 5 days at $4{ }^{\circ} \mathrm{C}$ and were $10-20$ $\mu \mathrm{m}$ in the longest dimension. Microcrystals for the experiment in June 2016 (run b) were prepared as above, but with an additional round of microseeding using crushed microcrystals to seed an additional round of microcrystal growth ${ }^{32}$. Micro-crystals were harvested by pipette and concentrated up to three-fold by centrifugation at $1000 \mathrm{~g}$ for $1 \mathrm{~min}$ followed by removal of supernatant. These crystals were somewhat thicker and, while diffracting to higherresolution, they highlighted the compromise inherent in TR-SFX since a lower excited-state occupancy was usually observed when working with crystals of higher optical density.

\section{Sample injection and data collection}

Microcrystals were transferred from Eppendorf tubes to a sample reservoir using a syringe and passing the microcrystal slurries through a stainless steel $20 \mu \mathrm{m}$ filter (VICI AG International) or a $20 \mu \mathrm{m}$ nylon filter (Sysmex). The reservoir was loaded into a temperature controlled rocking chamber and injected into the XFEL through a GDVN ${ }^{33}$ using an internal diameter of $75 \mu \mathrm{m}$. The microjet used a microcrystal suspension flow rate of $20 \mu 1 . \mathrm{min}^{-1}$ and was focused to a $10 \mu \mathrm{m}$ diameter using helium gas. The X-ray beam was aligned to interact 
467

468

469

470

471

472

473

474

475

476

477

478

479

480

481

482

483

484

485

486

487

488

489

490

491

with the liquid jet as close to the tip of the GDVN as practical and before Rayleigh breakup of the microjet.

Diffraction data were collected at $293 \mathrm{~K}$ at the CXI beam line ${ }^{34}$ of the LCLS XFEL during beamtime awarded in April 2015 (run a) and June 2016 (run b). Diffraction data were recorded on a Cornell-SLAC Pixel Array detector ${ }^{35}$. The X-ray wavelengths and equivalent pulse energies were $1.89 \AA(6.56 \mathrm{keV})$ in 2015 and $1.31 \AA(9.49 \mathrm{keV})$ in 2016 . An X-ray pulse duration of $36 \mathrm{fs}$ was used in 2015 and $45 \mathrm{fs}$ in 2016. The XFEL beam was focused to a $3 \mu \mathrm{m}^{2}$ spot for both experiments. The detector was located $89 \mathrm{~mm}$ from the microjet in 2015 and $145 \mathrm{~mm}$ from the microjet in 2016. Diffraction data were collected at a repetition rate of $120 \mathrm{~Hz}$ from microcrystals that were not exposed to any optical laser pump (dark-state) and for five time points corresponding to $\Delta t=1 \mathrm{ps}, 5 \mathrm{ps}, 20 \mathrm{ps}, 300 \mathrm{ps}$ and $8 \mu \mathrm{s}$ after photoexcitation. The time points $\Delta t=5 \mathrm{ps}$ and $300 \mathrm{ps}$ were repeated in both 2015 and 2016 and are referred to as data sets a and $\mathrm{b}$ respectively.

\section{Laser photoexcitation}

An optical Ti:Sa pump laser 150 fs in duration was focused into a spot size of $190 \mu \mathrm{m}$ FWHM $\left(323 \mu \mathrm{m} 1 / \mathrm{e}^{2}\right)$ and aligned to overlap with the LCLS X-ray pulse. The LCLS timing-tool ${ }^{36}$ provided a timing accuracy of $\pm 200 \mathrm{fs}$ for the time point, $\Delta t$, between the arrival of the optical pump laser and the X-ray probe. A pump-laser wavelength of $960 \mathrm{~nm}$ was used to photo-excite $\mathrm{RC}_{v i r}$ microcrystals, and this wavelength is at the absorption maximum of the special pair $\left(\varepsilon_{960} \approx 100000 \mathrm{M}^{-1} \cdot \mathrm{cm}^{-1}\right)$. The pump laser energy per pulse was $11.8 \mu \mathrm{J}$ in April 2015 and $11.0 \mu \mathrm{J}$ in June 2016. For an idealized Gaussian beam, $86.5 \%$ of this light will pass through a spot with diameter $1 / \mathrm{e}^{2}$ and $50 \%$ of this light will pass through a spot with diameter

FWHM. Thus the average fluence within the FWHM spot can be estimated as $25 \mathrm{~mJ} / \mathrm{cm}^{2}$ and 
$49223 \mathrm{~mJ} / \mathrm{cm}^{2}$ which equates to a pump-laser power-density of $138 \mathrm{GW} / \mathrm{cm}^{2}$ for the 2015

493 experiment and $129 \mathrm{GW} / \mathrm{cm}^{2}$ for the 2016 experiment. This calculation defines the units used

494 throughout to specify the laser power-density. Both values are above $30 \mathrm{GW} / \mathrm{cm}^{2}$ to 100

$495 \mathrm{GW} / \mathrm{cm}^{2}$ that has been recommended as an upper threshold to avoid nonlinear effects in 496 bacteriorhodopsin. ${ }^{37,38}$

Extreme non-linear absorption was observed as ultrafast sample heating in time-resolved X-

499 ray scattering studies of $\mathrm{RC}_{v i r}$ when pumped with $800 \mathrm{~nm}$ light. ${ }^{39}$ When using $800 \mathrm{~nm}$ to

500 photo-excite $\mathrm{RC}_{v i r}$ it is the $\mathrm{BCh}$ cofactors (rather than the $\mathrm{SP}$ ) which absorb light $\left(\varepsilon_{800} \approx\right.$

$\left.501180000 \mathrm{M}^{-1} \cdot \mathrm{cm}^{-1}\right)$. The pump-laser fluence used in that study ${ }^{39}$ was $1560 \mathrm{GW} / \mathrm{cm}^{2}$. Ultrafast

502 sample heating within a GDVN liquid microjet has also been measured as a function of the

$503800 \mathrm{~nm}$ pump-laser fluence using time-resolved X-ray scattering (Fig. 28 of reference ${ }^{40}$ ).

504 These measurements show that the energy deposited into $\mathrm{RC}_{v i r}$ samples is proportional to the

505 pump-laser fluence (a linear response) up to $270 \mathrm{GW} / \mathrm{cm}^{2}$ and that the measured heating then

506 varies quadratically (a non-linear response) above a pump-laser fluence of $355 \mathrm{GW} / \mathrm{cm}^{2}$. Thus

507 either an idealized assumptions of a perfectly aligned Gaussian beam may not be realistic,

508 and/or large losses occur as the incoming laser pulse is reflected from the surface of a GVDN

509 liquid microjet, and/or a thresholds $\mathrm{s}^{37,38}$ of $30 \mathrm{GW} / \mathrm{cm}^{2}$ to $100 \mathrm{GW} / \mathrm{cm}^{2}$ do not apply $\mathrm{RC}_{v i r}$

510 when photo-excited at $800 \mathrm{~nm}$. When $960 \mathrm{~nm}$ light is used to photo-excite the $\mathrm{SP}_{\text {of }} \mathrm{RC}_{v i r}$ it is

511 likely more difficult to induce non-linear effects because the photo-excited state SP* has an

512 absorption maximum red-shifted $70 \mathrm{~nm}$ relative to the ground state ${ }^{41}$ and hole-burning ${ }^{42}$ has

513 been observed in $\mathrm{RC}_{v i r}$ such that $\mathrm{SP}^{*}$ is effectively transparent to the incoming light.

514 Moreover, the absorbance of $\mathrm{RC}_{v i r}$ at $960 \mathrm{~nm}$ is only $56 \%$ of it absorbance at $800 \mathrm{~nm}$ and

515 therefore non-linear effects are likely to arise at higher power densities when using $960 \mathrm{~nm}$

516 rather than $800 \mathrm{~nm}$ to photo-excite $\mathrm{RC}_{v i r}$. Non-linear ultrafast heating ${ }^{40}$ is observed in $\mathrm{RC}_{v i r}$ 
517 delivered using a GDVN liquid microjet and photo-excited at $800 \mathrm{~nm}$ only above a power-

518 density of $355 \mathrm{GW} / \mathrm{cm}^{2}$. Therefore the $960 \mathrm{~nm}$ pump-laser power-densities of $138 \mathrm{GW} / \mathrm{cm}^{2}$

519 and $129 \mathrm{GW} / \mathrm{cm}^{2}$ used in this work are below where non-linear effects may reasonably be

520 anticipated. These conclusions are supported by time-resolved IR spectroscopy measurements

521 (Extended Data Fig. 1).

522

\section{Time-resolved infrared spectroscopy}

524 Time-resolved vibrational spectroscopy measurements were performed with a near infrared 525 (NIR) pump and mid-infrared (IR) probe setup using a regenerative amplifier (Spitfire Ace, 526 Spectra Physics) to deliver pulses centred at $800 \mathrm{~nm}(1.2 \mathrm{~mJ}, 5 \mathrm{kHz})$. The amplifier output is 527 used to pump a TOPAS-TWINS (Light Conversion) capable of generating tuneable 528 femtosecond pulses at two different wavelengths. One path was used to generate mid-infrared 529 probe light centred at $6000 \mathrm{~nm}$ via difference frequency generation whereas the other path 530 generated $960 \mathrm{~nm}$ pump pulses via second harmonic generation of the idler beam. The 960 $531 \mathrm{~nm}$ beam was chopped to $2.5 \mathrm{kHz}$ and delayed in time relative to the probe pulses using an optical delay line. Two weak replicas derived from the midIR beam were used as probe and corresponding reference. Both probe and reference were dispersed in a Horiba spectrograph (grating with $75 \mathrm{gr} / \mathrm{mm}$ ) and detected and integrated on a double-row MCT array with 64 pixels each on a shot-to-shot basis using a commercial detection system (Infrared Systems).

536 Samples of $\mathrm{RC}_{v i r}$ were prepared in a customized cell by enclosing ca. $15 \mathrm{uL}$ of solution $\left(\mathrm{RC}_{v i r}\right.$ 537 at ca. $0.4 \mathrm{mM}$ in $\mathrm{D}_{2} \mathrm{O}$ buffer) between two $2 \mathrm{~mm}$ thick $\mathrm{CaF}_{2}$ windows separated by a $25 \mu \mathrm{m}$ 538 spacer. Probe and reference beams were focused at the sample position and collimated using $53990^{\circ}$ off-axis parabolic mirrors. The pump beam was focused using a 30 -cm lens and 540 overlapped with the probe beam at its focus. The sample cell was placed where pump and 541 probe beams meet and translated continuously perpendicular to the beam direction during data 
acquisition. The focal spot size of the pump beam was determined using knife-edge scans and yielded perpendicular $1 / \mathrm{e}^{2}$ radii of $57 \mu \mathrm{m}$ and $56 \mu \mathrm{m}$. Different pump fluences were adjusted using reflective neutral density filters (Edmund Optics). For each fluence, twelve repeats over five time points (1000 pump shots per time point and repeat, at delays of -50, 1, 2, 5 and 300 ps) were recorded and less than 5\% of shots were rejected during data treatment. Signals were calculated by subtracting consecutive pump-on from pump-off shots followed by application of the noise reduction algorithm. ${ }^{43,44}$ The spectral resolution is $<5 \mathrm{~cm}^{-1}$. The results of these measurements are presented in Extended Data Fig. 1.

\section{Data processing}

Images containing more than 20 diffraction spots were identified as diffraction hits by Cheetah. ${ }^{45}$ Cheetah converted the raw detector data into the HDF5 format and data were then processed using the software suite CrystFEL version 0.6.2. ${ }^{46,47}$ Crystals were indexed using a tetragonal unit cell $\left(\mathrm{a}=\mathrm{b}=226.4 \AA, \mathrm{c}=113.7 \AA, \alpha=\beta=\gamma=90^{\circ}\right)$. Scaling and merging were performed using Monte Carlo methods using the same software. Data from the dark state and photo-excited states were scaled together using the custom dataset splitting option in the CrystFEL partialator module. Structure factors were calculated from merged intensities by the CCP4 module TRUNCATE ${ }^{48}$ and molecular replacement was performed using the CCP4 module Phaser ${ }^{49}$ using the ground-state $\mathrm{RC}_{\text {vir }}$ structures solved with $\mathrm{XFEL}$ radiation (PDB codes $5 \mathrm{O} 4 \mathrm{C}$ and $5 \mathrm{NJ} 4$ ) as a search models. Statistics for data collection and refinement are detailed in Extended Data Table 1.

\section{Electron density difference maps}

Isomorphous $\left|\mathrm{F}_{\text {obs }}\right|^{\text {light }}-\left|\mathrm{F}_{\text {obs }}\right|^{\text {dark }}$ difference Fourier electron density maps were calculated using the refined dark state structures for phases with the time-points $\Delta \underline{t}=5$ ps (data set a) and 300 
567 ps (data set a) calculated against data and coordinates using the pdb entry 5O4C whereas the 568 time point with the time-points $\Delta t=1 \mathrm{ps}, 5 \mathrm{ps}$ (data set b), $20 \mathrm{ps}, 300 \mathrm{ps}$ (data set b) and $8 \mu \mathrm{s}$ 569 were calculated against data and coordinates using the pdb entry $5 \mathrm{NJ} 4$. Thus all difference 570 electron density map calculations used only data collected during the same experiment. 571 Difference Fourier electron density maps represent measured changes in X-ray diffraction 572 intensities as changes in electron density without bias towards the photo-activated state's 573 structural model. The technique is extremely sensitive to small changes in electron density ${ }^{50}$ 574 and reveals more subtle features than are apparent from $2 \mathrm{mF}_{\text {obs }}-\mathrm{DF}_{\text {calc }}$ electron density maps 575 alone ( $\mathrm{m}$ is the figure of merit and $\mathrm{D}$ is estimated from coordinate errors). A Bayesian weighting calculation script ${ }^{51}$ using CNS software ${ }^{52}$ was also used to analyse the difference

577 Fourier electron density maps. In this procedure structure factor amplitude differences were 578 weighted by the product of the figure of merit of the ground state structure reflections and of a 579 weighting term, w (Equation 14 of reference $^{53}$ ), which was calculated 580 using Bayesian statistics developed to improve signal to noise ${ }^{53}$ For six of seven data-sets the 581 recurring difference electron density features were slightly strengthened by this step. The 582 exception was the time-point $\Delta t=8 \mu$ s which has difference electron density feature that are 583 weaker than for the other maps (Fig. 2f) and appears due to a lower occupancy of the charge 584 separated state in these microcrystals. It is possible that a fraction of the photo-oxidized $\mathrm{SP}^{-}$ 585 population is reduced from the C-subunit by $\Delta t=8 \mu \mathrm{s}$, which is longer than the time-scale of 586 this electron transfer step. ${ }^{26}$ However, no efforts were made to reduce the C-subunit when 587 preparing microcrystals and a similar occupancy $(30 \% \pm 5 \%)$ is observed to persist in time588 resolved spectroscopy measurements on crystals for up to millisecond delays. ${ }^{10}$ 589 
SVD analysis of difference Fourier electron density maps was performed using an in-house

592 code written in python that is based upon an approach previously described. ${ }^{54}$ As has been 593 discussed, ${ }^{55}$ SVD may serve as a noise-filter to enhance the signal across a sequence of 594 difference Fourier electron density maps. This step contains the assumption that the overall 595 mechanism is linear and that changes in electron density are similar over the selected timewindows. When applying SVD we evaluate the expression $[\mathrm{U}, \Sigma, \mathrm{V}]=\mathrm{SVD}(\mathrm{A})$, where $\mathrm{A}$ is a matrix of $n$ difference Fourier electron density maps containing $m$ elements; $U$ is an $n \times n$ unitary matrix; $\Sigma$ is an $\mathrm{n} \times \mathrm{m}$ rectangular matrix containing $\mathrm{n}$ diagonal elements (the singular values) arranged in decreasing order and all other matrix elements are zero; and the first right singular vector (the first row of the matrix V) is referred to as the principal component. Results from SVD analysis of all seven electron density maps are presented in Fig. 2e and Extended Data Fig. 31,m. Results from SVD analysis deriving from the first four time-points $(\Delta t=1 \mathrm{ps}, 5 \mathrm{ps}$ a and b, $20 \mathrm{ps})$ and the last three time-points $(\Delta t=300 \mathrm{ps}$ a and $\mathrm{b}, 8 \mu \mathrm{s})$ are shown in Fig. 3c,d; Extended Data Fig. 3h,i; Extended Data Fig. 5h,i; Supplementary Videos 1 and 2. This separation of the maps is motivated by the fact that photo-activated $\mathrm{RC}_{v i r}$ molecules have menaquinone oxidized for the first sub-set of time-points yet most menaquinone molecules of photo-activated $\mathrm{RC}_{v i r}$ are reduced for the second sub-set of timepoints.

Structural refinement of photo-excited states

611 Isomorphous $\left|\mathrm{F}_{\mathrm{obs}}\right|^{\text {light }}-\left|\mathrm{F}_{\mathrm{obs}}\right|^{\text {dark }}$ difference Fourier electron density maps were inspected in 612 COOT. Structural refinement was performed using Phenix. ${ }^{56}$ A model was first placed within 613 the unit cell using rigid body refinement followed by multiple rounds of partial-occupancy 614 refinement where the $\mathrm{SP}, \mathrm{BCh}_{\mathrm{L}}, \mathrm{BPh}_{\mathrm{L}}, \mathrm{Q}_{\mathrm{A}}$, portions of $\mathrm{TM}$ helices $\mathrm{E}_{\mathrm{L}}, \mathrm{D}_{\mathrm{L}}, \mathrm{E}_{\mathrm{M}}$ and $\mathrm{D}_{\mathrm{M}}$, as well 615 as connecting loops, and additional residues near cofactors (L153-178, L190, L230, L236- 

248, M193-221, M232, M243-253, M257-266) were allowed to adopt a second conformation 617 with $30 \%$ occupancy and the dark-state structure (pdb entry 5O4C) was held fixed. The occupancy of $30 \%$ was chosen by assessing the results from partial occupancy refinement when the occupancy was allowed to vary and was imposed for all structural refinements for consistency. Results from structural refinement were compared against the difference electron densities and some manual adjustments were made using COOT. ${ }^{57}$ Refinement statistics are displayed in Extended Data Table 1. Validation of structure geometry was performed using MOLPROBITY ${ }^{58}$ and PROCHECK. ${ }^{59}$ Structural changes were also validated by calculating simulated difference Fourier electron density maps from the refined structures ${ }^{10,20}$ (Extended Data Figs. 21 and 4j).

626

\section{Structural analysis of large-scale protein motions}

The high multiplicity of SFX data was exploited for structural analysis by randomly selecting a sub-set of experimental observations from within each SFX data-set to create 100 separate 630 (but not independent) serial crystallography data-sets for the two resting state data-sets and the seven photo-activated data-sets, amounting to 900 resampled data-sets in total. For each of these resampled data-sets the mean and uncertainty estimates $(\sigma)$ for every unique Bragg reflection were determined. Structural refinement over a cycle of 100 rigid body and 100 recovered. Coordinate errors associated with each individual structural refinement are estimated $^{60}$ to be $\leq 0.2 \AA$.

639 
640 The distances between the $\mathrm{C} \alpha$ atoms of the photo-activated and resting $\mathrm{RC}_{v i r}$ structures were 641 compared pairwise using the miller package of CCTBX. ${ }^{61}$ A $100 \times 100$ Euclidian distance 642 matrix was then calculated for every $\mathrm{C} \alpha$ atom and every time point according to: $\Delta \mathrm{r}_{\mathrm{ij}}{ }^{\Delta t \text {,dark }}=$ $643\left|\mathbf{r}_{\mathrm{i}}^{\Delta t}-\mathbf{r}_{\mathrm{j}}^{\text {dark }}\right|$, where $i$ and $j$ vary from 1 to100 and denote resampled dataset numbers, $\Delta \mathrm{r}_{i, j}$ 644 depicts the distance separating the C $\alpha$ coordinates of datasets $i$ and $j$, and $\mathbf{r}_{\mathrm{i}}^{\Delta t}$ and $\mathbf{r}_{\mathrm{j}}^{\text {dark }}$ are the 645 refined coordinates obtained from the photo-activated or dark structures, respectively. A 646 second order Taylor series expansion was then used to estimate the mean and error associated 647 with the ratio $\Delta \mathrm{r}_{\mathrm{ij}}^{\Delta t, \text { dark }} / \Delta \mathrm{r}_{\mathrm{ij}}{ }^{\text {dark,dark }}$ arising from coordinate variations within each set of 100 648 structural refinements. This expansion leads to the expression: predictions (Fig. 4d-f, Supplementary Video 4).

662 
664 For each of the seven experimental difference Fourier electron density maps $(\Delta t=1 \mathrm{ps}, 5 \mathrm{ps}$ 665 (data set a), 5 ps (data set b), 20 ps, 300 ps (data set b), 300 ps (data set a), $8 \mu$ s) a lower666 pedestal of $3.0 \sigma$ was applied such that all electron density with an amplitude $<3.0 \sigma$ was set 667 to zero. Both positive and negative difference electron densities were then integrated within a $6684.5 \AA$ radius sphere about a chosen coordinate (Extended Data Fig. 3j) as described for the 669 analysis of TR-SFX data recorded from bacteriorhodopsin. ${ }^{20}$ These positive (A+) and 670 negative (A-) integrated difference electron density amplitudes were merged to yield a single amplitude according to: $\mathrm{A}(\mathbf{r})=\left((\mathrm{A}+)^{2}+(\mathrm{A}-)^{2}\right)^{1 / 2}$ about the centre of integration $\mathbf{r}$. The results of this analysis are presented in Fig. $2 \mathrm{f}$ where six centres of integration, $\mathbf{r}$, are chosen as: the centre of the $\mathrm{BPh}_{\mathrm{M}}$ ring; the magnesium atom of $\mathrm{BCh}_{\mathrm{M}}$; the mid-point between the two magnesium atoms of the two SP bacteriochlorophylls; the magnesium atom of $\mathrm{BCh}_{\mathrm{L}}$; the centre of the $\mathrm{BPh}_{\mathrm{L}}$ ring; and the centre of the ketone containing six-carbon ring of menaquinone $\mathrm{Q}_{\mathrm{A}}$.

For tests of statistical significance (Extended Data Table 3), this set was complemented by the addition of amplitudes extracted by integration about the iron atoms of haem 1, hame $_{2}$, haem 3 and haem 4 to create a set of ten amplitudes for each of the seven time points:

$$
\left[\mathrm{A}\left(\mathrm{BPh}_{\mathrm{M}}\right), \mathrm{A}\left(\mathrm{BCh} \mathrm{M}_{\mathrm{M}}\right), \mathrm{A}(\mathrm{SP}), \mathrm{A}\left(\mathrm{BCh}_{\mathrm{L}}\right), \mathrm{A}\left(\mathrm{BPh}_{\mathrm{L}}\right), \mathrm{A}\left(\mathrm{Q}_{\mathrm{A}}\right), \mathrm{A}\left(\mathrm{H}_{1}\right), \mathrm{A}\left(\mathrm{H}_{2}\right), \mathrm{A}\left(\mathrm{H}_{3}\right), \mathrm{A}\left(\mathrm{H}_{4}\right)\right]_{\Delta t}
$$
arranged as a $10 \times 7$ element matrix. Control "noise only" $\left|\mathrm{F}_{\mathrm{obs}}\right|^{\text {dark }}-\left|\mathrm{F}_{\mathrm{obs}}\right|^{\text {dark }}$ isomorphous difference Fourier electron density maps were calculated by first selecting sixteen resampled data-sets from the set of 100 generated from the $2015 \mathrm{RC}_{v i r}$ dark data, and sixteen resampled data-sets from the set of 100 generated from the $2016 \mathrm{RC}_{v i r}$ dark data. Eight $\left|\mathrm{F}_{\text {obs }}\right|^{\text {dark }}-\left|\mathrm{F}_{\text {obs }}\right|^{\text {dark }}$ isomorphous difference Fourier electron density maps were then calculated by pair-wise comparisons between the sixteen resampled data-sets of the 2015 data, and another eight difference Fourier electron density maps were calculated by pairwise-comparisons of the 
sixteen resampled data-sets of the 2016 data. Seven control difference Fourier electron density maps were then randomly selected from the set of sixteen "noise-only" maps, difference electron density values with an amplitude lower than $3 \sigma$ were set to zero, and a set of $[\mathrm{A}(\mathbf{r}$,dark-dark $)]$ were created by integrating the remaining difference electron density within a $4.5 \AA$ radius sphere centred upon the $\mathrm{RC}_{v i r}$ cofactors as described above. A two-sample $t$ test was then performed in MATLAB to determine if the set of seven time-dependent amplitudes $[\mathrm{A}(\mathbf{r}, \Delta t)]$ and the set of seven "noise-only" amplitudes $[\mathrm{A}(\mathbf{r}$,dark-dark $)]$ were indistinguishable from one another (the null hypothesis). The $t$-tests were then repeated 1000 times by randomly selecting a different combination of seven control amplitudes [A(r,darkdark)] from the sixteen "noise-only" difference Fourier electron density maps calculated above (of $16 ! /(9 ! \times 7 !)=11440$ possible different combinations of the 16 control maps). The results of this analysis are summarized in Extended Data Table 3 and show that, when a threshold of $p \leq 0.001$ is applied, the difference electron density amplitudes associated with the SP cannot be ascribed to noise. When a threshold of $p \leq 0.0125$ is applied and the last three time-points $(\Delta t=300 \mathrm{ps}(\mathrm{a}), 300 \mathrm{ps}(\mathrm{b}), 8 \mu \mathrm{s})$ are examined as a set, the difference electron density amplitudes associated with the $\mathrm{SP}, \mathrm{BCh}_{\mathrm{L}}$ and $\mathrm{Q}_{\mathrm{A}}$ cannot be ascribed to noise. Conversely, the set of difference electron density amplitudes associated with most other cofactors, as well as all sets of difference electron density amplitudes generated from noise-only maps, are indistinguishable from noise according to the results of this two sample $t$-test 708 (Extended Data Table 3).

711 Initial coordinates were taken from PDB databank entry 5O4C and missing residues and co712 factor segments were retrieved from PDB entry 1PRC. ${ }^{62}$ Protonation states of residues were 713 chosen based on their reference $\mathrm{pK}_{\mathrm{a}}$ values and structural criteria such as hydrogen bond 
714 interactions. After the addition of protons to the structure, a 200 step steepest descent

715 geometry optimization was performed with Gromacs $4.5^{63}$ to relax these coordinates. During

716 this optimization the positions of the heavy atoms were constrained to their positions in the $\mathrm{x}$ -

717 ray structure. As in previous work, the interactions were modelled with the Amber03 force-

718 field. ${ }^{64,65}$ Non-bonded Coulomb and Lennard-Jones interactions were evaluated without

719 periodic boundary conditions (PBC) and using infinite cut-offs.

721 After relaxing hydrogens with molecular mechanics (MM) optimization, we performed 722 several Quantum Mechanics (QM)/MM geometry optimizations of all atoms in the reaction 723 centre, using the interface between the TeraChem quantum chemistry package ${ }^{66,67}$ and 724 Gromacs 4.5. ${ }^{63}$ These optimizations were also performed without PBC and with infinite cut725 offs for the Coulomb and Lennard-Jones interactions. The QM subsystems (Extended Data 726 Fig. 6a-c) were modelled with unrestricted DFT. In these DFT calculation we used the PBE0 727 functional $^{68}$ in combination with the LANL2DZ basis set. ${ }^{69}$ Empirical corrections to 728 dispersion energies and interactions were introduced with Grimme's DFT-D3 model. ${ }^{70}$ The 729 remainder of the protein, including crystal water molecules, was modelled with the Amber03 730 force-field, ${ }^{64,65}$ in combination with the TIP3P water model. ${ }^{71}$ We searched for minimum731 energy geometries in all relevant oxidation states of the system using the limited-memory 732 Broyden-Fletcher-Goldfard-Shannon quasi-Newton optimization algorithm.

734 The goal of these optimization steps was to characterize the structural relaxation of the protein 735 in response to changes in the electronic states of the cofactors along the A-branch of the 736 photo-induced electron transfer process. We therefore examined the following electronic 737 configurations:

738 i. All cofactors in their resting states: $\mathrm{SP}^{0}, \mathrm{BPh}_{\mathrm{L}}{ }^{0}, \mathrm{Q}_{\mathrm{A}}{ }^{0}$ 
ii. Special pair photo-excited, other cofactors in resting state: $\mathrm{SP}^{*}, \mathrm{BPh}_{\mathrm{L}}{ }^{0}, \mathrm{Q}_{\mathrm{A}}{ }^{0}$

740 iii. Special pair photo-oxidized, $\mathrm{BPh}_{\mathrm{L}}$ reduced: $\mathrm{SP}^{+1}, \mathrm{BPh}_{\mathrm{L}}{ }^{-1}, \mathrm{Q}_{\mathrm{A}}{ }^{0}$

741 iv. Special pair photo-oxidized, $\mathrm{Q}_{\mathrm{A}}$ reduced: $\mathrm{SP}^{+1}, \mathrm{BPh}_{\mathrm{L}}{ }^{0}, \mathrm{Q}_{\mathrm{A}}{ }^{-1}$

742 Since including all co-factors into one large QM-region is computationally too demanding, we 743 performed the optimizations with a different QM subsystem for each co-factor, including nearest residues, in all relevant electronic states. The structural response to the change in electronic state (Fig. 4d-f) was obtained by comparing the optimized geometries and potential energies in the various oxidation states.

To quantify the effect of photo-absorption by the special pair (SP, i to ii) we first optimized the resting state with the SP plus nearby residues in the QM region (Extended Data Fig. 6a), described at the PBE0/LANL2DZ level of theory plus D3 dispersion corrections. This structure was used as a reference for the optimized structures in the excited state (SP*, ii) and after photo-oxidation $\left(\mathrm{SP}^{+1}\right.$, iii). Using the same $\mathrm{QM} / \mathrm{MM}$ subdivision, we optimized the system in the first singlet excited state $\left(S_{1}\right)$ by switching the QM description to the Timedependent DFT within the Tamm-Dancoff approximation, ${ }^{72}$ and in the photo-oxidized state by switching the spin state of the electronic wave function to the lowest energy doublet state $\left(\mathrm{D}_{0}\right)$. In the $\mathrm{QM} / \mathrm{MM}$ optimization of the $\mathrm{D}_{0}$ state of the $\mathrm{SP}$, we modelled the $\mathrm{BPh}_{\mathrm{L}}$ with point charges representing the reduced state of that co-factor. Only very modest protein structural changes were associated with the optimized geometries with the SP in the $\mathrm{S}_{1}$ and $\mathrm{D}_{0}$ relative to the reference structure in the resting state $\left(\mathrm{S}_{0}\right)$. Likewise, we also optimized the geometry of the protein with the $\mathrm{BPh}_{\mathrm{L}}$ and nearby residues in the QM region (Extended Data Fig. 6d) in both the lowest energy singlet $\left(\mathrm{S}_{0}, \mathrm{i}\right)$ and doublet $\left(\mathrm{D}_{0}\right.$, reduced, iii) states. In the optimization 
763 very similar (Extended Data Fig. 6e).

765

766

767

768

\section{Stabilization energies}

oxidized $\left(\mathrm{D}_{0}, \mathrm{SP}^{+1}\right)$ state. Again the structural response is rather minor, as the geometries are

In the next step of the electron transfer process, the electron transfers from $\mathrm{BPh}_{\mathrm{L}}$ to $\mathrm{Q}_{\mathrm{A}}$ (iv). We optimized the protein with $\mathrm{Q}_{\mathrm{A}}$ plus its immediate environment, including the non-heme $\mathrm{Fe}^{2+}$ site, in the QM region. The optimized structures in the resting and reduced states are compared in Extended Data Fig. 6f. Reduction of $\mathrm{Q}_{\mathrm{A}}$ from menaquinone to (deprotonated) semiquinone induces significant structural changes in the $\mathrm{Q}_{\mathrm{A}}$ binding pocket. In line with the difference densities observed at 300 ps after photo-excitation, the hydrogen bond between the QA carbonyl and His $217_{\mathrm{M}}$ reduces by $0.17 \AA$. We suggest that the reduction of this hydrogen bond helps stabilizing the negative charge on the $\mathrm{Q}_{\mathrm{A}}$.

To quantify the overall structural response to the electron transfers, we computed the displacements of the atoms in the various states (ii-iv) with respect to the structure of resting state (i) and recorded these displacements as B-factors to the pdb coordinate file of the resting state. Because only one co-factor was included in the QM region of our QM/MM optimizations, we summed up the displacements of both QM/MM optimizations of each redox state. Fig. 4d-f represents these displacements as colours.

To estimate the energetic effects of the protein structural changes on the electron transfer process, we computed the adiabatic and vertical electron affinities for $\mathrm{Q}_{\mathrm{A}}$ in isolation and in the optimized QM/MM protein models. These energies are shown schematically in Extended Data Fig. 6g,h. For the neutral $\mathrm{Q}_{\mathrm{A}}$ in vacuum, the electron affinity without structural relaxation is $164.5 \mathrm{~kJ} \mathrm{~mol}^{-1}$ (vertical electron affinity, VEA). Structural relaxation in response 
788 to adding the electron increases the affinity further by $24 \mathrm{~kJ} \mathrm{~mol}^{-1}$, so that the energy 789 difference between the neutral reactant minimum on the one hand and the reduced product 790 minimum is $188.5 \mathrm{~kJ} \mathrm{~mol}^{-1}$ (adiabatic electron affinity, AEA) on the other hand. The 791 calculated AEA is in good agreement with results from previous computations, ${ }^{29}$ but is an 792 overestimation with respect to the experimental value for the related 1,4-naphthoquinone (175 $\left.793 \mathrm{~kJ} \cdot \mathrm{mol}^{-1}\right){ }^{73}$ Inside the protein environment, the VEA is much higher $\left(258 \mathrm{~kJ} . \mathrm{mol}^{-1}\right)$, part of 794 which we attribute to the electrostatic interaction between the reduced $\mathrm{Q}_{\mathrm{A}}$ co-factor with the 795 positively charged $\mathrm{Fe}^{2+}$ ligand site. Structural relaxation of both the $\mathrm{Q}_{\mathrm{A}}$ cofactor and the 796 protein environment increases the electron affinity by $60 \mathrm{~kJ}^{\mathrm{mol}}{ }^{-1}$ to yield an AEA of 318 $797 \mathrm{~kJ} \cdot \mathrm{mol}^{-1}$. Thus, the results of the computations suggest that the structural response of the 798 protein adds another $36 \mathrm{~kJ} \cdot \mathrm{mol}^{-1}$ to the intrinsic relaxation energy of $\mathrm{Q}_{\mathrm{A}}\left(24 \mathrm{~kJ} \cdot \mathrm{mol}^{-1}\right.$ in 799 vacuum) as concluded in earlier computations. ${ }^{29}$ We note that in this analysis we focussed 800 only on the effect of the structural response on the affinity of $\mathrm{Q}_{\mathrm{A}}$. To estimate the total 801 reaction energy associated with the photo-induced electron transfer process from the SP to $802 \mathrm{Q}_{\mathrm{A}}$, we also need the absolute energies of the neutral, photo-excited and oxidized states of the $803 \mathrm{SP}$ as well as the neutral and reduced states of $\mathrm{BPh}_{\mathrm{L}}$. However, since these energies were not 804 computed with identical QM/MM setups, we do not provide an accurate estimate here. 


\section{METHODS REFERENCES}

810

81131 Wöhri, A. B. et al. Lipidic sponge phase crystal structure of a photosynthetic reaction 812 center reveals lipids on the protein surface. Biochemistry 48, 9831-9838 (2009).

81332 Dods, R. et al. From Macrocrystals to Microcrystals: A Strategy for Membrane 814 Protein Serial Crystallography. Structure 25, 1461-1468 (2017).

81533 DePonte, D. P. et al. Gas dynamic virtual nozzle for generation of microscopic droplet $816 \quad$ streams. J. Phys. D: Appl. Phys. 41, 195505 (2008).

81734 Liang, M. et al. The Coherent X-ray Imaging instrument at the Linac Coherent Light $818 \quad$ Source. J Synchrotron Radiat 22, 514-519 (2015).

81935 Hart, P. et al. The CSPAD megapixel x-ray camera at LCLS. Proc. SPIE 8504C, $820 \quad 85040 \mathrm{C} \quad 85011(2012)$.

82136 Harmand, M. et al. Achieving few-femtosecond time-sorting at hard X-ray free$822 \quad$ electron lasers. Nature Phot. 7, 215-218 (2013).

82337 Nass Kovacs, G. et al. Three-dimensional view of ultrafast dynamics in photoexcited $824 \quad$ bacteriorhodopsin. Nat Commun 10, 3177 (2019).

82538 Miller, R. J. D., Pare-Labrosse, O., Sarracini, A. \& Besaw, J. E. Three-dimensional 826 view of ultrafast dynamics in photoexcited bacteriorhodopsin in the multiphoton 827 regime and biological relevance. Nat Commun 11, 1240 (2020).

82839 Arnlund, D. et al. Visualizing a protein quake with time-resolved X-ray scattering at a 829 free-electron laser. Nat Methods 11, 923-926 (2014).

83040 Arnlund, D. X-ray free-electron laser based methods for structural and ultrafast 831 dynamics studies of a photosynthetic reaction centre $\mathrm{PhD}$ thesis, University of 832 Gothenburg, (2014). 
83341 Fleming, G. R., Martin, J. L. \& Breton, J. Rates of primary electron transfer in 834 photosynthetic reaction centres and their mechanistic implications. Nature 333, 190$835192(1988)$.

83642 Meech, S. R., Hoff, A. J. \& Wiersma, D. A. Role of charge-transfer states in bacterial 837 photosynthesis. Proc Natl Acad Sci U S A 83, 9464-9468 (1986).

83843 Feng, Y., Vinogradov, I. \& Ge, N. H. General noise suppression scheme with 839 reference detection in heterodyne nonlinear spectroscopy. Opt Express 25, 26262$840 \quad 26279(2017)$.

84144 Feng, Y., Vinogradov, I. \& Ge, N. H. Optimized noise reduction scheme for 842 heterodyne spectroscopy using array detectors. Opt Express 27, 20323-20346 (2019).

84345 Barty, A. et al. Cheetah: software for high-throughput reduction and analysis of serial 844 femtosecond X-ray diffraction data. J. Appl. Crystallogr. 47, 1118-1131 (2014).

84546 White, T. A. et al. CrystFEL: a software suite for snapshot serial crystallography. $J$. $846 \quad$ Appl. Crystallogr. 45, 335-341 (2012).

84747 White, T. A. et al. Recent developments in CrystFEL. J. Appl. Crystallogr. 49, 680$848 \quad 689(2016)$.

84948 French, S. \& Wilson, K. Treatment of Negative Intensity Observations. Acta $850 \quad$ Crystallogr A 34, 517-525 (1978).

85149 McCoy, A. J. et al. Phaser crystallographic software. J. Appl. Crystallogr. 40, 658-674 $852 \quad$ (2007).

85350 Henderson, R. \& Moffat, J. K. The Ditferenee Fourier Technique in Protein 854 Crystallography: Errors and their Treatment. Acta Crystallogr. B 27, 1414-1420 $855 \quad$ (1971).

85651 Wickstrand, C., Dods, R., Royant, A. \& Neutze, R. Bacteriorhodopsin: Would the real $857 \quad$ structural intermediates please stand up? Biochim Biophys Acta 1850, 536-553 (2015). 
85852 Brunger, A. T. et al. Crystallography \& NMR system: A new software suite for 859 macromolecular structure determination. Acta Crystallogr D Biol Crystallogr 54, 905$860921(1998)$.

86153 Ursby, T. \& Bourgeois, D. Improved Estimation of Structure-Factor Difference 862 Amplitudesfrom Poorly Accurate Data. Acta Crystallogr. A. 53, 564-575 (1997).

86354 Rajagopal, S., Schmidt, M., Anderson, S., Ihee, H. \& Moffat, K. Analysis of 864 experimental time-resolved crystallographic data by singular value decomposition. 865 Acta Crystallogr D Biol Crystallogr 60, 860-871 (2004).

86655 Schmidt, M., Rajagopal, S., Ren, Z. \& Moffat, K. Application of singular value 867 decomposition to the analysis of time-resolved macromolecular x-ray data. Biophys $J$ $868 \quad \mathbf{8 4}, 2112-2129(2003)$.

86956 Adams, P. D. et al. PHENIX: a comprehensive Python-based system for 870 macromolecular structure solution. Acta Crystallogr. D Biol. Crystallogr. 66, 213-221 $871 \quad(2010)$.

87257 Emsley, P. \& Cowtan, K. Coot: model-building tools for molecular graphics. Acta 873 Crystallogr. D Biol. Crystallogr. 60, 2126-2132 (2004).

87458 Chen, V. B. et al. MolProbity: all-atom structure validation for macromolecular 875 crystallography. Acta Crystallogr. D Biol. Crystallogr. 66, 12-21 (2010).

87659 Laskowski, R. A., MacArthur, M. W., Moss, D. S. \& Thornton, J. M. PROCHECK: a 877 program to check the stereochemical quality of protein structures. J. Appl. Cryst. 26, $878 \quad 283-291(1993)$.

87960 Murshudov, G. N. \& Dodson, E. J. Simplified error estimation a la Cruickshank in 880 macromolecular crystallography. CCP4 Newsletter on protein crystallography 33, 31881 39 (1997). 
88261 Grosse-Kunstleve, R. W., Sauter, N. K., Moriarty, N. W. \& Adams, P. D. The 883 Computational Crystallography Toolbox: crystallographic algorithms in a reusable $884 \quad$ software framework. J. Appl. Cryst. 35, 126-136 (2002).

88562 Deisenhofer, J., Epp, O., Sinning, I. \& Michel, H. Crystallographic refinement at 2.3 886 A resolution and refined model of the photosynthetic reaction centre from 887 Rhodopseudomonas viridis. J. Mol. Biol. 246, 429-457 (1995).

88863 Pronk, S. et al. GROMACS 4.5: a high-throughput and highly parallel open source 889 molecular simulation toolkit. Bioinformatics 29, 845-854 (2013).

89064 Duan, Y. et al. A point-charge force field for molecular mechanics simulations of 891 proteins based on condensed-phase quantum mechanical calculations. J. Comput. $892 \quad$ Chem. 24, 1999-2012 (2003).

89365 Ceccarelli, M., Procacci, P. \& Marchi, M. An ab initio force field for the cofactors of 894 bacterial photosynthesis. J. Comput. Chem. 24, 129-142 (2003).

89566 Ufimtsev, I. S. \& Martinez, T. J. Quantum Chemistry on Graphical Processing Units. 896 3. Analytical Energy Gradients, Geometry Optimization, and First Principles 897 Molecular Dynamics. J Chem Theory Comput 5, 2619-2628 (2009).

89867 Titov, A. V., Ufimtsev, I. S., Luehr, N. \& Martinez, T. J. Generating Efficient 899 Quantum Chemistry Codes for Novel Architectures. J Chem Theory Comput 9, 213$900221(2013)$.

90168 Adamo, C. \& Barone, V. Toward Reliable Density Functional Methods without 902 Adjustable Parameters: The PBE0 Model. J. Chem. Phys. 110, 6158-6170. (1999).

90369 Dunning T.H. \& P.J., H. in Methods of Electronic Structure Theory. Modern 904 Theoretical Chemistry, Vol. 3 (ed Schaefer H.F.) (Springer, 1977). 
90570 Grimme, S., Antony, J., Ehrlich, S. \& Krieg, H. A consistent and accurate ab initio 906 parametrization of density functional dispersion correction (DFT-D) for the 94 907 elements H-Pu. J Chem Phys 132, 154104-154101 - 154104-154119 (2010).

90871 Jorgensen, W. L., Chandrasekhar, J., Madura, J. D., Impey, R. W. \& Klein, M. L. 909 Comparison of simple potential functions for simulating liquid water. J. Chem. Phys. $910 \quad 79,926-935(1983)$.

$91172 \quad$ Hirata, S. \& Head-Gordon, M. Time-Dependent Density Functional Theory within the 912 Tamm-Dancoff Approximation. Chem. Phys. Lett. 314, 291-299 (1999).

91373 Heinis, T., Chowdhury, S., Scott, S. L. \& Kebarle, P. Electron Affinities of Benzo-, 914 Naphtho-, and Anthraquinones Determined from Gas-Phase Equilibria Measurements. 915 J. Am. Chem. Soc. 110, 400-407 (1988).

91674 Maiti, S. et al. Femtosecond coherent transient infrared spectroscopy of reaction 917 centers from Rhodobacter sphaeroides. Proc Natl Acad Sci U S A 91, 10360-10364 $918 \quad$ (1994).

91975 Pawlowicz, N. P. et al. Identification of the first steps in charge separation in bacterial 920 photosynthetic reaction centers of Rhodobacter sphaeroides by ultrafast mid-infrared 921 spectroscopy: electron transfer and protein dynamics. Biophys J 95, 1268-1284 (2008).

922

923

924

925

926 
Extended Data Figure 1: Dependence of transient infrared (IR) spectra on the pump

laser fluence. a, Time-resolved IR difference spectra recorded from $\mathrm{RC}_{v i r}$ in $\mathrm{D}_{2} \mathrm{O}$ buffer for

931 four time points and variable pump-laser fluence following $960 \mathrm{~nm}$ excitation. Spectral

932 changes are consistent with earlier reports. ${ }^{74,75}$ Dominant time-dependent features are 933 highlighted and include: (1) a negative band at $1687 \mathrm{~cm}^{-1}$ (9-carbonyl stretch in $\mathrm{SP}_{\mathrm{L}}$ and $\mathrm{SP}_{\mathrm{M}}$ ) 934 and (2) a broad negative band centred at $1680 \mathrm{~cm}^{-1}$ (9-carbonyl stretch in $\left.\mathrm{BCh}_{\mathrm{L}}\right)$. Transient 935 changes from $\Delta t=2$ ps to 5 ps correlate with the time-dependent photo-oxidation of SP. Grey 936 columns indicate decreasing bands whereas cyan columns indicate increasing bands. b, Time 937 resolve IR difference spectra normalized and superimposed. These spectra remain 938 superimposable throughout the pump-laser fluence domain probed. c, Dependence of the 939 magnitude of the difference IR signal (calculated as the sum of the absolute value over all 940 pixels) on the pump laser fluence. Below $20 \mathrm{GW} / \mathrm{cm}^{2}$ the absolute signal increases 941 approximately linearly whereas above $60 \mathrm{GW} / \mathrm{cm}^{2}$ the signal flattens out as a plateau is 942 reached. This plateau is consistent with the complete bleaching of the special pair absorption 943 at $960 \mathrm{~nm}$ in the photoexcited state and correlates with the phenomenon of hole-burning in $944 \quad \mathrm{RC}_{v i r}{ }^{42}$

946 Extended Data Figure 2: Overview of the experimental $F_{\text {obs }}{ }^{\text {light }}-\mathbf{F}_{\text {obs }}{ }^{\text {dark }}$ difference 947 Fourier electron density maps calculated between the photo-excited and resting state 948 data. a, Overview of the structure of $\mathrm{RC}_{v i r}$ when viewed from the plane of the membrane. $\mathrm{TM}$ 949 helices $E_{L}$ and $D_{M}$ are highlighted in red and gold respectively. b-h, Difference Fourier 950 electron density maps for the time-points: $\mathbf{b}, \Delta t=1 \mathrm{ps} ; \mathbf{c}, \Delta t=5 \mathrm{ps}(\mathrm{a}) ; \mathbf{d}, \Delta t=5 \mathrm{ps}(\mathrm{b}) ; \mathbf{e}, \Delta t$ $951=20 \mathrm{ps} ; \mathbf{f}, \Delta t=300 \mathrm{ps}(\mathrm{b}) ; \mathbf{g}, \Delta t=300 \mathrm{ps}(\mathrm{a}) ; \mathbf{h}, \Delta t=8 \mu \mathrm{s}$. All maps are contoured at $\pm 4.0 \sigma$ 
952 ( $\sigma$ is the root mean square electron density of the map). Positive difference electron density is

953 shown in blue and negative difference electron density is shown in gold.

954

Extended Data Figure 3: Light-induced electron density changes in $\mathrm{RC}_{v i r}$ at the site of

photo-oxidation. Experimental $\mathrm{F}_{\mathrm{obs}}^{\text {light }}-\mathrm{F}_{\mathrm{obs}}$ dark isomorphous difference Fourier electron density maps for the time-points: a, $\Delta t=1 \mathrm{ps} . \mathbf{b}, \Delta t=5 \mathrm{ps}$ (data set a); $\mathbf{c}, \Delta t=5$ ps (data set b). d, $\Delta t=20 \mathrm{ps} ; \mathbf{e}, \Delta t=300 \mathrm{ps}$ (data set b); f, $\Delta t=300 \mathrm{ps}$ (data set a); g, $\Delta t=8 \mu \mathrm{s}$. h-i, Principal component from SVD analysis of difference Fourier electron density maps for: $\mathbf{h}$, the first four time-points, $\Delta \mathrm{t}=1 \mathrm{ps}, 5 \mathrm{ps}$ (data set a), 5 ps (data set b) and $20 \mathrm{ps}$; i, the final three time-points, $\Delta \mathrm{t}=300 \mathrm{ps}$ (data set a), $300 \mathrm{ps}$ (data set b) and $8 \mu \mathrm{s}$. All maps are contoured at $\pm 3.2 \sigma$ (blue, positive difference electron density; gold, negative difference electron density). Peak heights listed in Table 2 are marked with coloured circles in panels $\mathrm{h}$ and i. j, Representation of a sphere of radius $4.5 \AA$ used to integrate difference electron density above a pedestal of $3.0 \sigma$ as described in reference ${ }^{20}$. Positive difference density amplitudes $(\mathrm{A}+)$ and negative difference density amplitudes (A-) were merged according to $967\left((\mathrm{~A}+)^{2}+(\mathrm{A}-)^{2}\right)^{1 / 2}$ and are displayed in Fig. 2f. These integrated difference electron density values are also used in the statistical analyses presented in Extended Data Table 2. k, Simulated difference Fourier electron density maps near the SP calculated from the refined structure for $\Delta t=20 \mathrm{ps}$ versus the refined resting state structure. Data are cut at $2.8 \AA$ resolution for comparison with experimental data. The simulated map is contoured at \pm 12.0 $\sigma . \mathbf{l}$, Relative magnitude of the singular values resulting from SVD analysis of the difference Fourier electron density maps for all seven time-points. m, First (blue) and second (red) columns of the unitary matrix $U$ resulting from SVD analysis of all seven time-points weighted according to their corresponding singular values. The first right singular vector (the 976 principal SVD component) makes a significant and positive contribution to all time-points. In 
contrast the difference electron density maps for $\Delta t=5 \mathrm{ps}$ (a) and $300 \mathrm{ps}$ (a) contain strong negative contributions from the second right singular vector, which suggests that differences between the two experimental runs cause systematic differences in the difference Fourier electron density maps that are separated by SVD analysis.

Extended Data Figure 4: Electron density changes near the active site of myoglobin. The $\mathrm{F}_{\mathrm{obs}}{ }^{\text {light }}-\mathrm{F}_{\mathrm{obs}}{ }^{\text {dark }}$ difference Fourier electron density map for $\Delta t=10 \mathrm{ps}$ was calculated from data (pdb entries 5CNE minus 5CMV) recorded during TR-SFX studies of the photodissociation of carbon monoxide from the active site of myoglobin. ${ }^{21} \mathbf{a}$, Data from $15 \AA$ to 1.8 $\AA$ were used to calculate the difference Fourier electron density map. b, Data from $15 \AA$ to 3.0 $\AA$ were used to calculate the difference Fourier electron density map. Positive and negative difference electron density features associated with the heme group indicate slight motions of the protoporphyrin-IX. Blue represents positive difference density (contoured at $3.0 \sigma$ ) and gold represents negative density (contoured at $-3.0 \sigma$ ). At $1.8 \AA$ the maximum amplitude of the highlighted difference density features are: $\mathrm{a}^{+}, 14.5 \sigma ; \mathrm{a}-, 14.0 \sigma ; \mathrm{b}^{+}, 4.6 \sigma ; \mathrm{b}-4.0 \sigma ; \mathrm{c}^{+}$, $4.1 \sigma, \mathrm{c}-4.0 \sigma ; \mathrm{d}+, 3.8 \sigma$. When the map is recalculated after data is cut to $3.0 \AA$ resolution the corresponding values are: $\mathrm{a}+, 11.9 \sigma ; \mathrm{a}-, 12.4 \sigma ; \mathrm{b}+, 4.2 \sigma ; \mathrm{b}-4.1 \sigma ; \mathrm{c}+, 3.7 \sigma$, c- $2.9 \sigma ; \mathrm{d}+, 3.2$ $\sigma$.

\section{Extended Data Figure 5: Light-induced electron density changes in $\mathrm{RC}_{v i r}$ within the} menaquinone binding pocket. Experimental $\mathrm{F}_{\mathrm{obs}}{ }^{\text {light }}-\mathrm{F}_{\mathrm{obs}}{ }^{\text {dark }}$ difference Fourier electron density maps for the time-points: $\mathbf{a}, \Delta t=1 \mathrm{ps} . \mathbf{b}, \Delta t=5 \mathrm{ps}$ (data set a); $\mathbf{c}, \Delta t=5 \mathrm{ps}$ (data set b). d, $\Delta t=20 \mathrm{ps} ; \mathbf{e}, \Delta t=300 \mathrm{ps}($ data set b); f, $\Delta t=300 \mathrm{ps}$ (data set a); $\mathbf{g}, \Delta t=8 \mu \mathrm{s}$. All seven maps are contoured at $\pm 3.0 \sigma$ (blue, positive difference electron density; gold, negative difference electron density). h-i, Principal component from SVD analysis of difference 
1002 Fourier electron density maps for: $\mathbf{h}$, the first four time-points, $\Delta \mathrm{t}=1 \mathrm{ps}, 5 \mathrm{ps}$ (data set a), $5 \mathrm{ps}$

1003 (data set b) and $20 \mathrm{ps}$; i, the final three time-points, $\Delta \mathrm{t}=300 \mathrm{ps}$ (data set a), $300 \mathrm{ps}$ (data set b)

1004 and $8 \mu$ s. SVD difference Fourier maps are contoured at $\pm 3.2 \sigma$. Peak heights listed in Table

10052 are indicated in panel i. j, Simulated difference Fourier electron density maps near $\mathrm{Q}_{\mathrm{A}}$

1006 calculated from the refined structure for $\Delta t=300 \mathrm{ps}$ (data set a) versus the refined resting

1007 state structure. Data are cut at $2.8 \AA$ resolution for comparison with experimental data. The

1008 simulated map is contoured at $\pm 12.0 \sigma$.

1009

1010 Extended Data Figure 6: Results of QM/MM energy minimization calculations. a-c, QM

1011 subsystems used in the QM/MM optimizations of the co-factors. Atoms included in the QM

1012 region are shown in ball-and-stick representation, while the other atoms of the protein are

1013 shown as cartoons. Atoms belonging to the co-factor that are not included in the QM region

1014 are shown as sticks. Water molecules that are not part of the QM region are not shown. a,

1015 Atoms included in the QM region associated with the SP. b, Atoms included in the QM

1016 region associated with the $\mathrm{BPh}_{\mathrm{L}}$. c, Atoms included in the $\mathrm{QM}$ region associated with the $\mathrm{Q}_{\mathrm{A}}$.

1017 d, Structures of the energy minimized resting conformation (black: $\mathrm{SP}$ and $\mathrm{BPh}_{\mathrm{L}}$ neutral) and

1018 that after the first electron transfer step (blue: $\mathrm{SP}^{+1}$ and $\mathrm{BPh}_{\mathrm{L}}^{-1}$ ) shown near the special pair. e,

1019 Structures of the energy minimized resting conformation (black: SP and $\mathrm{BPh}_{\mathrm{L}}$ neutral) and

1020 that after the first electron transfer step (blue: $\mathrm{SP}^{+1}$ and $\mathrm{BPh}_{\mathrm{L}}^{-1}$ ) shown near $\mathrm{BPh}_{\mathrm{L}}$. f, Structures

1021 of the energy minimized resting conformation (black: SP and $\mathrm{Q}_{\mathrm{A}}$ neutral) and that after the

1022 second electron transfer step (blue: $\mathrm{SP}^{+}$and $\mathrm{Q}_{\mathrm{A}}{ }^{-}$) shown near $\mathrm{Q}_{\mathrm{A}}$. The H-bond between $\mathrm{O}_{1}$ of

1023 the reduced semiquinone and $\mathrm{N}_{\delta 1}$ of His $217_{\mathrm{M}}$ is predicted to be shortened by $0.17 \AA$ when

1024 menaquinone is reduced. g-h, Schematic depiction of the potential energy surfaces for

1025 menaquinone in vacuum $(\mathbf{g})$ and within the protein $(\mathbf{h})$ in the resting (neutral) electronic state

1026 (black) and the reduced electronic state (red). Vertical electron affinities (VEA), adiabatic 
1027 electron affinities (AEA) and relaxation energies $E_{r}$ were computed at the PBE01028 D3/LANL2DZ level of DFT for isolated $\mathrm{Q}_{\mathrm{A}}$ and at the PBE0-D3/LANL2DZ/Amber03 1029 QM/MM level for $\mathrm{Q}_{\mathrm{A}}$ within the protein.

1030

1031 Extended Data Figure 7: Recurring movements of $\mathrm{C} \alpha$ atoms quantified by structural 1032 refinement. Recurring movements of $\mathrm{C} \alpha$ atoms quantified by full occupancy structural 1033 refinement against 100 randomly resampled TR-SFX data sets. Recurring movements are 1034 represented as error weighted mean ratios relative to 100 control structural refinements 1035 (Methods). Error weighted means ratios are coloured from grey $(<80 \%$ of the maximum 1036 ratio) to red ( $\geq 95 \%$ of the maximum ratio). a, Recurring movements of $\mathrm{C} \alpha$ atoms associated 1037 with refinements against data for $\Delta t=1 \mathrm{ps}$. $\mathbf{b}$, Recurring movements of $\mathrm{C} \alpha$ atoms for $\Delta t=5$ 1038 ps (data set a). c, Recurring movements of $\mathrm{C} \alpha$ atoms for $\Delta t=5 \mathrm{ps}$ (data set b). d, Recurring 1039 movements of $\mathrm{C} \alpha$ atoms for $\Delta t=20 \mathrm{ps}$. e, Recurring movements of $\mathrm{C} \alpha$ atoms for $\Delta t=300 \mathrm{ps}$ 1040 (data set b). f, Recurring movements of $\mathrm{C} \alpha$ atoms for $\Delta t=300 \mathrm{ps}$ (data set a). g, Recurring 1041 movements of $\mathrm{C} \alpha$ atoms for $\Delta t=8 \mu \mathrm{s}$. Transmembrane helices are drawn as rods.

1042

1043 
Extended Data Table 1: Crystallographic data and refinement statistics

1047 Footnotes to Extended Data Table 1

1048

1049

1050

1051

1052

1053

1054

1055

1056

1057

1058

1059

1060

1061

1062

1063

1064

1065

1066

1067

1068

1069

1070

1071

1072

1073

1074

1075

1076

1077

1078

1079

1080

${ }^{\dagger} \mathrm{R}_{\text {split }}=1 / \sqrt{2 \frac{\sum h k l \mid \text { Ieven-Iodd } \mid}{1 / 2 \sum h k l \mid \text { Ieven }+ \text { Iodd } \mid}}$

Values in parenthesis is those of the highest resolution shell.

${ }^{£}$ Ratio of the number of indexed images to the total number of images.

\section{Extended Data Table 2: Difference Fourier electron density peak amplitudes}

Footnotes to Extended Data Table 2

* Positive and negative difference electron density peaks are marked on Extended data Figs. $3 \mathrm{~h}, 3 \mathrm{i}$ and $5 \mathrm{i}$. Difference electron density values were read out manually in COOT. Amplitudes $<2.4 \sigma$ are not shown.

\section{Extended Data Table 3: Two sample t-tests of integrated electron density amplitudes}

Footnotes to Extended Data Table 3

*Mean $\sigma$ /sphere represents the root-mean-square of the difference electron density above and below a threshold of $\pm 3.0 \sigma$ within a sphere of radius $4.5 \AA$ centred on the $\mathrm{Mg}^{2+}$ atoms of the $\mathrm{BChs}$; the $\mathrm{Fe}$ atoms of the haems; and ring centres of the menaquinone and BPhe cofactors. All values are scaled relative to a mean value for $\mathrm{SP}=1$.

${ }^{\dagger}$ The number of elements in each sampled set of the two-sample t-test.

${ }^{\ddagger}$ Difference electron density associated with haem 3 gave the lowest mean $\sigma /$ sphere $(0.14)$ and an exceptionally low standard deviation $( \pm 0.06)$. Randomly generated control data gave the corresponding values of $0.09 \pm 0.22$. The coincidence of a set with a low standard deviation being compared against a control set with an exceptionally high standard deviation frequently yielded low $p$-values. Nevertheless, the experimental difference features associated with haem 3 are weak and are therefore not physically meaningful.

${ }^{\mathfrak{f}}$ Thirty two control data sets were generated by randomly selecting a sub-set of the dark observations to generate a new data set. Sixteen control difference Fourier electron density maps were then calculated between two of the control data-sets to generate maps representing the noise inherent within the SFX experiment. Mean $\sigma /$ sphere calculations and all other steps proceeded as with the light versus dark experimental difference Fourier electron density maps.

${ }^{\epsilon}$ The reference set consisted of a set of seven maps $\left(\mathrm{N}_{\mathrm{B}}=7\right)$ randomly selected from sixteen control maps. Random selection of the control set and the t-test was repeated 1000 
1081 times, from a possible set of $16 ! /(9 ! \times 7 !)=11440$ different combinations of the 16 control 1082 maps. Percentage values give the fraction of occasions when $p \leq$ threshold for the resulting 1083 two sample t-test.

$1084{ }^{\$}$ The hypothesis that the two sets of the t-test are indistinguishable is either true $(0)$ or false 1085 (1) at the specified confidence level.

$1086 \S$ Features identified as distinguishable from noise with a degree of confidence defined by 1087 the given $p$-value.

1088 


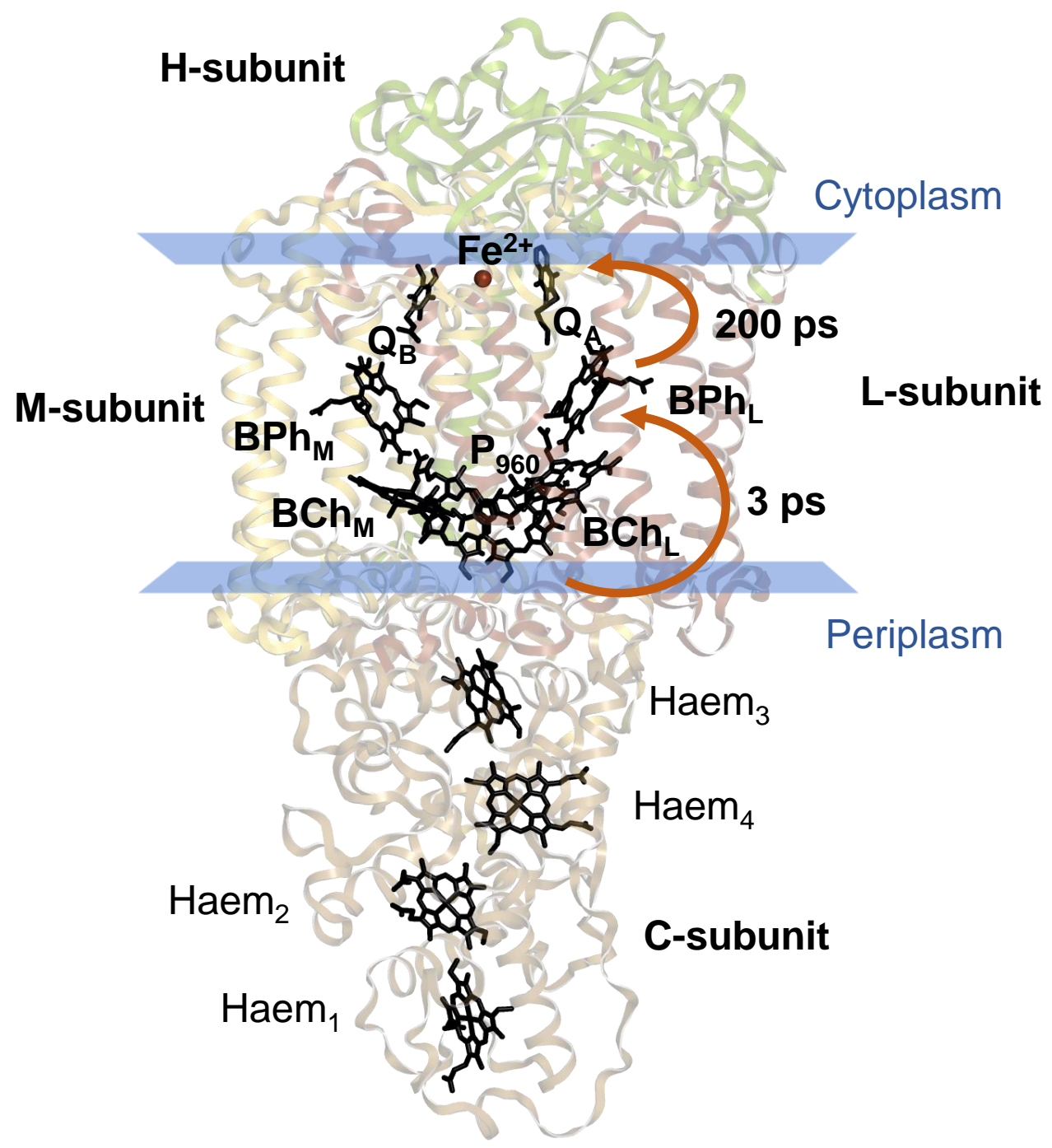

Figure 1: Dods et al. 

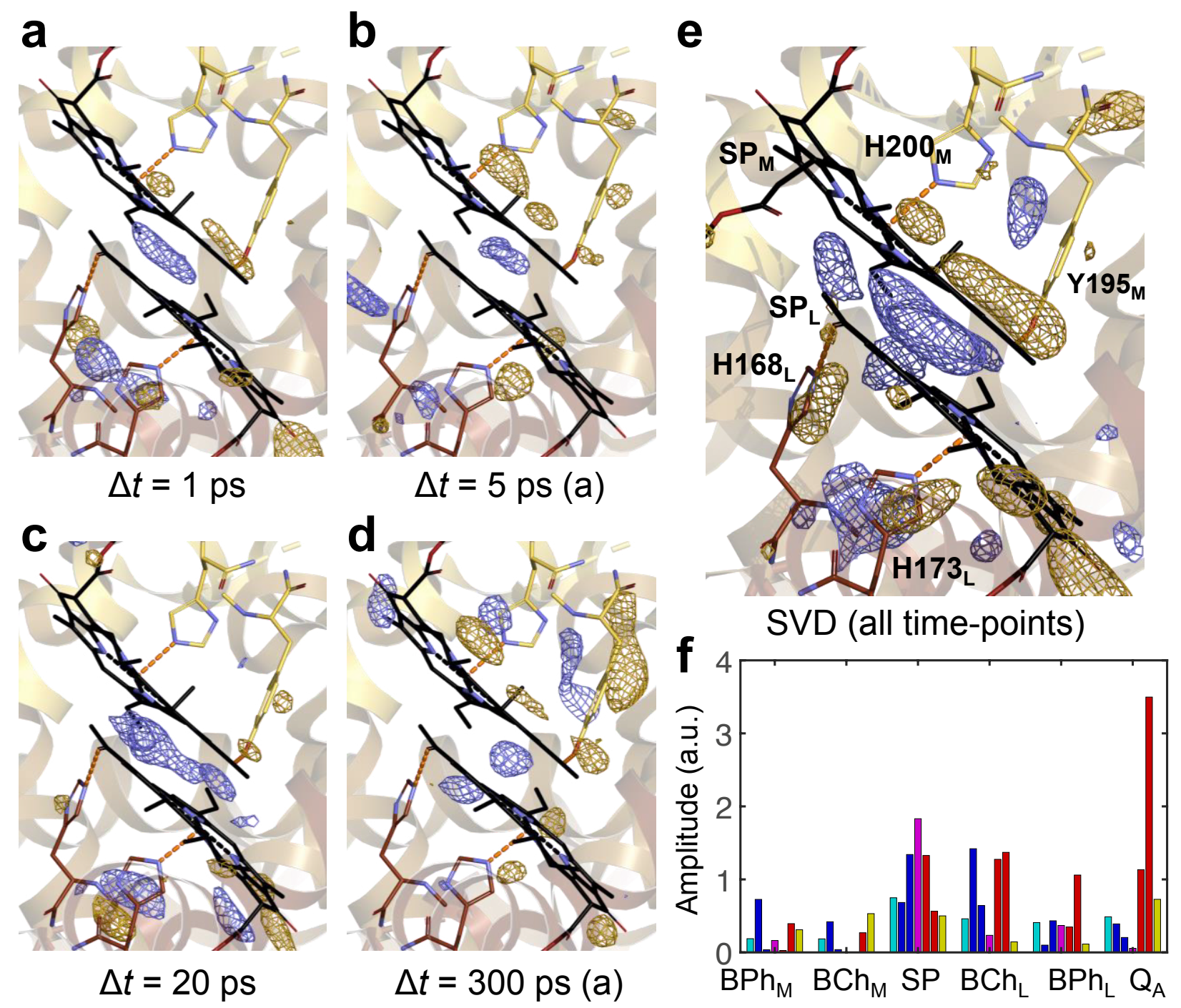

Figure 2: Dods et al. 
a

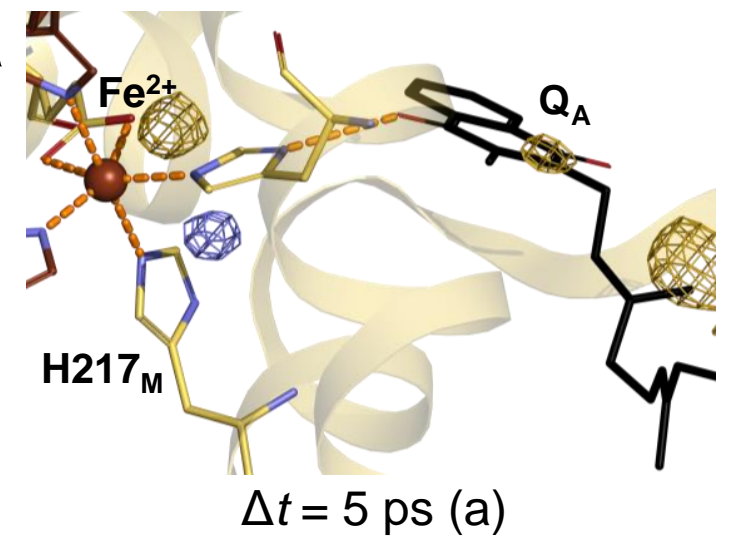

c

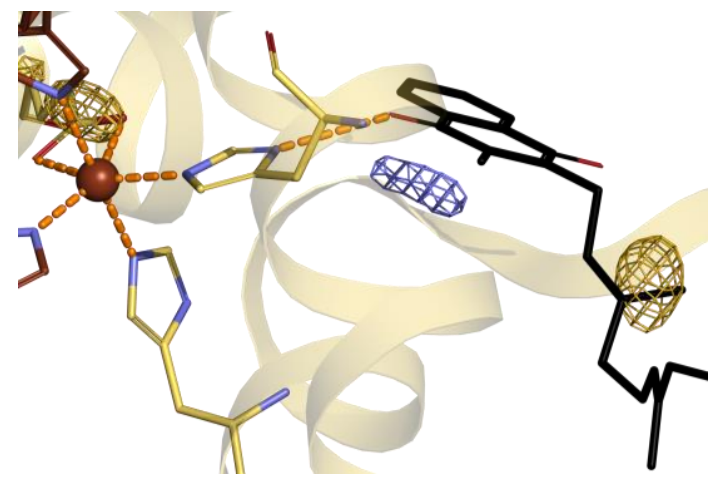

SVD (1 ps, $5 \mathrm{ps}$ (a \& b), $20 \mathrm{ps}$ )

e

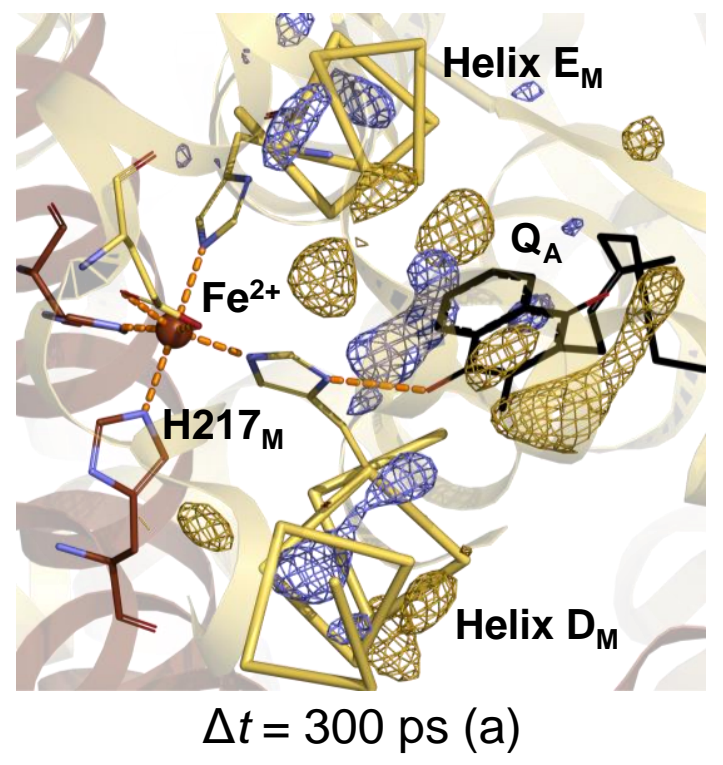

b

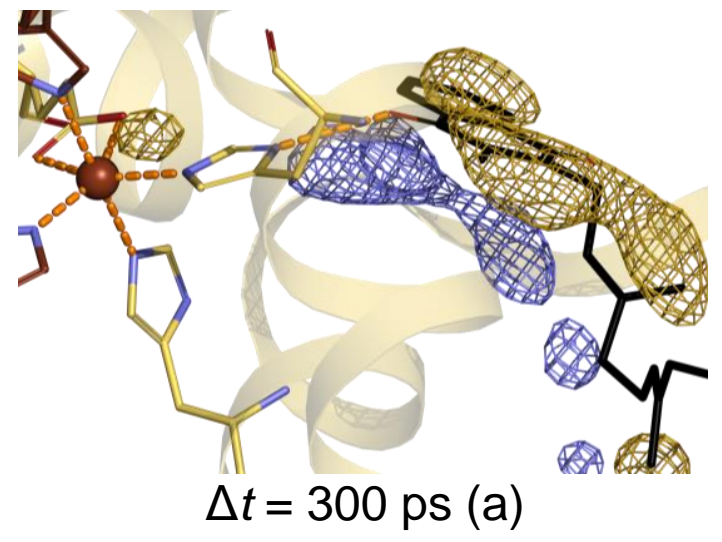

d

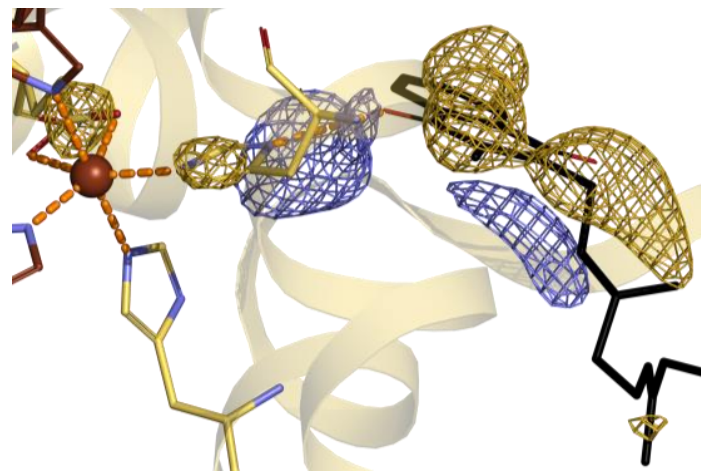

$\operatorname{SVD}(300$ ps (a \& b), $8 \mu \mathrm{s})$

f

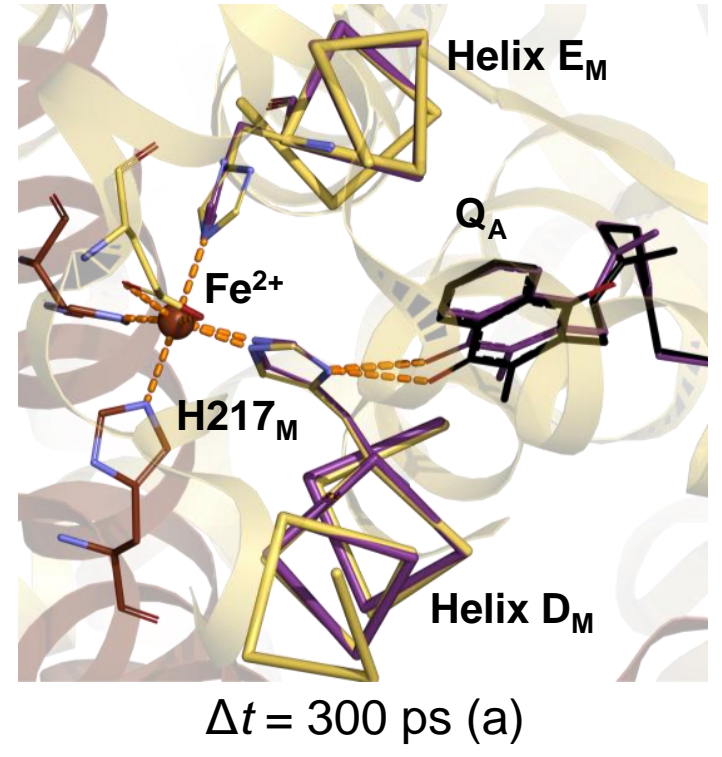

Figure 3: Dods et al. 

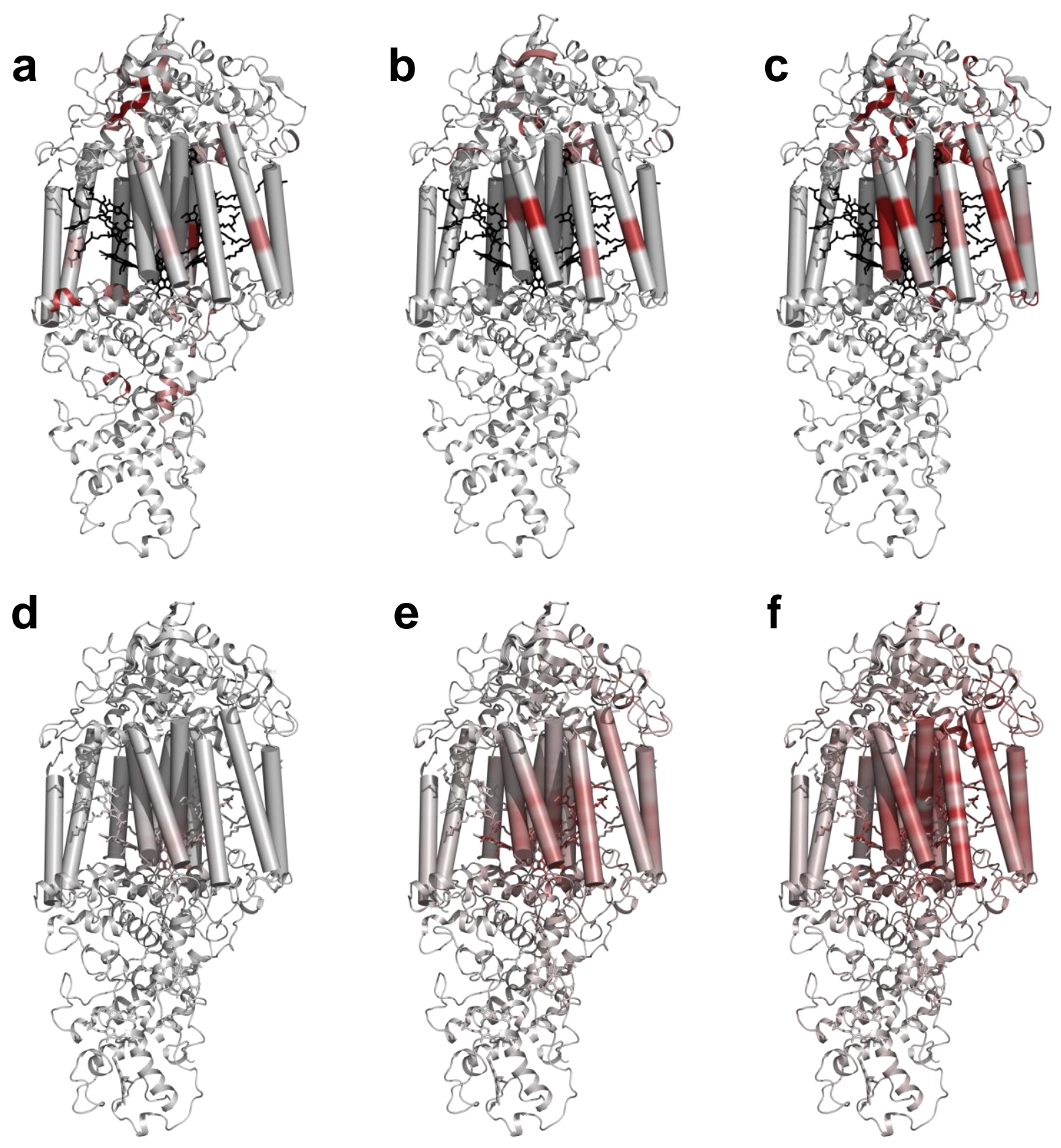

Figure 4: Dods et al. 